\title{
A nonlinear circular ring model with rotating effects for tire vibrations
}

\author{
Trong Dai $\mathrm{Vu}^{\mathrm{a}}$, Denis Duhamel ${ }^{\mathrm{a}, *}$, Zouhir Abbadi ${ }^{\mathrm{b}}$, Hai-Ping Yin ${ }^{\mathrm{a}}$, Arnaud \\ Gaudin $^{\mathrm{b}}$ \\ ${ }^{a}$ Université Paris-Est, Laboratoire Navier, ENPC/IFSTTAR/CNRS, \\ 6 et 8 Avenue Blaise Pascal, \\ Cité Descartes, Champs sur Marne, \\ 77455 Marne la Vallée, cedex 2, France \\ ${ }^{b}$ PSA Peugeot Citroën, \\ Automotive Research and Advanced Engineering Division, \\ Centre Technique de Vélizy A, \\ Route de Gisy - cc vv1415, \\ 78943 Vélizy-Villacoublay, France
}

\begin{abstract}
Rolling noise contributes significantly to the noise inside cars. This noise comes from the tire/road contact and for low frequencies $(0-400 \mathrm{~Hz})$, it is mainly transmitted into the cabin through structural vibrations. Thus estimating this noise requires modelling the tire vibrations by taking into account the rotating effects and the contact with rough surfaces. Concerning the model of rolling tire, a formulation of a deformable solid is constructed by using an Arbitrary Lagrangian Eulerian approach. This formulation is applied on a new simplified tire model which is a circular ring including shear stresses and nonlinear effects due to the vehicle load. This model is successfully validated by comparison with FEM results.
\end{abstract}

Keywords: rolling noise, vibrations, tire/road contact, structural dynamics, rotating effects, gyroscopic effects, circular ring model

\footnotetext{
${ }^{*}$ Corresponding author

Email addresses: vutro@enpc.fr (Trong Dai Vu), denis.duhamel@enpc.fr (Denis Duhamel), zouhir.abbadi@mpsa.com (Zouhir Abbadi), hp@lami.enpc.fr (Hai-Ping Yin), arnaud.gaudin@mpsa.com (Arnaud Gaudin)
} 


\section{Introduction}

The interior noise of vehicles has an important source coming from tires in the low frequency range, typically up to $400 \mathrm{~Hz}$. For these frequencies the rolling noise is mainly due to tire vibrations. These vibrations are themselves created by the unsteady contact between road asperities and the tire tread pattern. For predicting this noise generation an accurate tire model is necessary. In the past, various tire models have been developed focusing on different aspects of the problem.

A first class of models is the two and three-dimensional circular ring models. For instance Böhm [1], Heckl [2] and Kropp [3] have modelled the tread as a circular Euler-Bernoulli beam. Sidewalls are represented by radial and tangential springs. This model takes into account the effect of the internal pressure and is linear. These circular ring models are very useful for analysing the radial vibrations of tires for low frequencies. In 2000, Périsse [4] compared the computation of the velocity of a point on the ring with its experimental value and showed a good agreement at low frequencies (below $400 \mathrm{~Hz}$ ). Several authors developed circular ring models by adding the effect of rotation, see for instance Meftah [5], Périsse [6] and Campanac [7, 8]. In this case one can reproduce the phenomenon of splitting of modes depending on the rotational speed. This property is also found in the work of Kim and Bolton [9]. In addition, Huang [10] has analysed the rotating ring model under a suspension system.

So, two-dimensional circular ring models allow the modelling of the dynamic behaviour of tires for low frequencies [0-400Hz]. Analytical transfer functions can be obtained to deal with contact problems. To use these models, it is necessary to identify some parameters (stiffness of the spring, bending stiffness ...). However, these two-dimensional circular ring models do not allow observing out of plane modes (toggle mode). That is why Eichler [11] and Gipser [12] enriched a circular model by adding a lateral stiffness to the tread and a model of rigid rim. All these models are linear and do not allow to consider, for instance, the influence of the vehicle load or nonlinear material behaviours on tire vibrations.

For higher frequencies, one needs other approaches. In 2003, Muggleton [13] modelled a tire using two orthotropic plates in traction. The tread is represented by a plate and the sidewalls are replaced by two plates. Hamet [14] has analysed the vibrations of a tire under a point force applied at the centre of the plate. This allows the construction of Green's functions of the tire used to treat the contact problem. Both models of Hamet [14] and Muggleton [13] do not take into account the effect of the curvature of the tire. Another model with two plates has been developed by Pinnington $[15,16,17]$. His model takes into account the curvature of the tire. The effect of the curvature of the tire is to couple the radial and circumferential movements and normal and tangential forces in all the vibratory responses of 
the tire. The dynamic equations of the tread are written for one-dimensional waves propagating around the belt and a standing wave through the tread. The effects of curvature, shear stiffness, inertia of rotation, stress, rotation velocity and the air pressure are included. The two-dimensional Mindlin plate is used to formulate this model. It is applicable to a wide band of frequencies $[0-3 \mathrm{kHz}]$. The difficulty of the model is still the determination of the parameters of the tread and the sidewalls. Kim and Bolton [18] have also proposed a model of rotating cylindrical shell. Similarly, based on the analytical model developed by Kropp, Wullens and Kropp [19] analysed the vibration field of a tire and the Doppler effect due to the rotation.

Because of the complexity of the tire geometry and the heterogeneous materials from which it is made, an analytical model cannot completely describe the vibrations of the tire. Moreover, the coupling between the air cavity and the tread is difficult to describe analytically. Therefore it is necessary to build numerical models of the whole set, tire / wheel / cavity, for predicting the vibratory responses of a tire. For example, one can cite the models of Takagi [20], Narasimha [21], Richards [22] and Cho [23]. Taking into account the different materials of the layers of the tire, Takagi [20] used two-dimensional finite elements. The behaviour of materials is assumed linear and the tire pressure and the rotation are taken into account. This model is valid in the frequency range $[0-250 \mathrm{~Hz}]$.

Regarding the three-dimensional numerical models, Fadavi [24] and Brinkmeier [25] used Abaqus to model a tire. The properties of the model are identified by experimental measurements. The carcass and sidewalls are described by transverse isotropic materials. The rubber of the pads is described by a hyper-elastic material and the contact with the road is taken into account. This model is valid up to $1500 \mathrm{~Hz}$. Narasimha [21] also modelled a smooth tire in contact with an obstacle and Richards [22] modelled the tire air cavity. The coupling between the fluid and the structure is included in the model. So by comparing with measurements, one sees that the model is valid up to $400 \mathrm{~Hz}$. Another possibility is to use waveguides as in Waki [26] or Duhamel [27, 28]. In this case only a small section of the tire needs to be computed which saves a lot of computational resources for medium and high frequencies. More precisely, concerning the coupling with the fluid, one has to consider the coupling with the internal fluid in the cavity between the tire and the wheel for loaded or unloaded tires leading to first resonances slightly above $200 \mathrm{~Hz}$ as measured and modelled for instance in $[29,30,31]$. This point will not be considered in this article as it has been well studied in these articles. Similarly no coupling with the external fluid will be considered, as this point has also be well studied in the past.

Often, authors have neglected the quasi-static deformation of tires and confused the stationary configuration with the configuration of reference (the configuration of reference is the initial configuration which undergoes a rigid rotation and the stationary configuration is that of the quasi-stationary 
regime wherein all material points deform statically under the effects of exterior loads). Under the load of the vehicle and the effect of the air pressure, the tire undergoes a nonlinear deformation. It is thus necessary to distinguish the deformed and non-deformed configurations. The deformed configuration is illustrated by the image of a crushed tire. This configuration is not known. It is obtained by solving the equilibrium equations in stationary regime. This point was for instance developed by [32, 33, 34] which used a FEM model for computing the response of tires including the quasi-static deformation and gyroscopic effects. However, these FEM models leads to heavy computations.

This paper aims at developing a nonlinear ring model to estimate the influence of these nonlinearities with a low computational cost. A first step towards the solution of this problem is a good understanding of the nonlinear behaviour of beams. In this domain, Antman and al. [35, 36, 37, 38, 39, $40,41,42,43]$ have established general formulations for the behaviour of beams. Simo $[44,45,46]$ described the problem of nonlinear beams with large rotations in three dimensions. These authors have also proposed a finite element method to solve the problem. Davi [47] took the Kirchhoff hypothesis to build equilibrium relationships for a thin beam. He therefore neglected the deformation due to shear stress. Applying these results Lanzo $[48,42]$ has analysed the stability of a multi-layer model of a rubber beam and the influence of a large shear force on the nonlinear deformation of a beam.

From these ideas, we develop here a nonlinear circular ring model with a good representation of shear deformations to get a mostly analytical model able to estimate the influence of non linearities. Note that we focus on the behaviour of the tire and not on the solution of the global problem of a tire rolling on a real road. So the contact with the road is not in the scope of this article. The structure of the article is the following. In section two the general equations for bodies with finite rotation and deformation are described. In section three a Timoshenko beam model including shear and rotating effects with large deformations is considered. Finally, in section four, examples and validations are given before a conclusion.

\section{Finite rotation and transformation of deformable solids}

A study of the dynamics of a deformable solid in finite transformation and rotation requires different configurations. Two states should be considered:

- a stationary state: This is the configuration in which the deformed solid is static. It is obtained from the crushed configuration by applying a rigid rotation. This means that the displacements of the material points depend only on their positions. 
- a dynamic state: It is a small perturbation around the stationary state. This disturbance is time dependent.

The Arbitrary Lagrangian Eulerian (ALE) approach [49] is valid to treat the stationary motion. For the dynamic movement, formulations on a selected reference configuration will be developed. This selected reference configuration could be the rigidly rotating configuration or the stationary deformed configuration depending on the way that the problem is set in the following.

\subsection{Equations of motion}

In this section, the different configurations with the different movements are defined. Then, the equilibrium equations of each deformed state are built.
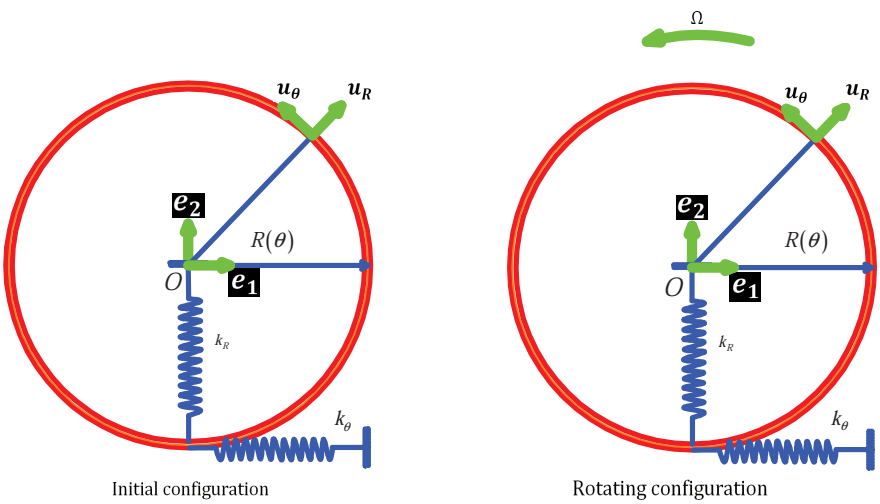

Rotating configuration
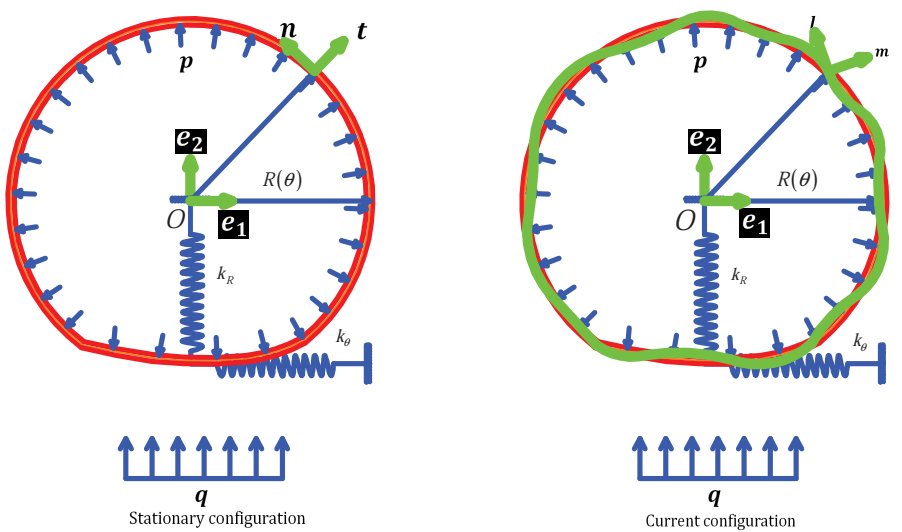

Figure 1: Description of the different configurations 
In Fig 1, the four possible configurations of the solid during the deformation are presented: The initial configuration denoted $D_{0}$, the rotating configuration denoted $D_{r}$, the stationary configuration denoted $D_{s}$ and the vibrating (or current) configuration denoted $D_{t}$. A point in the physical space is represented by its coordinates $\left(X_{1}, X_{2}, X_{3}\right)$ in a fixed orthonormal reference system $\mathfrak{R}\left(O, \mathbf{e}_{1}, \mathbf{e}_{2}, \mathbf{e}_{3}\right)$. Quantities with indices $(.)_{0},(.)_{r},(.)_{s},(.)_{t}$ are relative to the configurations $D_{0}, D_{r}, D_{s}$ and $D_{t}$ respectively. So the subscript 0 refers to the initial configuration, the subscript $r$ to the rigidly rotating configuration, the subscript $s$ to the stationary deformed configuration and the subscript $t$ to the final current configuration as defined in Fig 1. For instance, a material point $M$ is:

- $M_{0}$ in $D_{0}$. So that we can write: $\mathbf{X}_{0}=\mathbf{O M}_{0}=X_{0_{1}} \mathbf{e}_{1}+X_{0_{2}} \mathbf{e}_{2}+X_{0_{3}} \mathbf{e}_{3}$

- $M_{r}$ in $D_{r}: \mathbf{X}_{r}=\mathbf{O M}_{r}=X_{r_{1}} \mathbf{e}_{1}+X_{r_{2}} \mathbf{e}_{2}+X_{r_{3}} \mathbf{e}_{3}$

- $M_{s}$ in $D_{s}: \mathbf{X}_{s}=\mathbf{O M}_{s}=X_{s_{1}} \mathbf{e}_{1}+X_{s_{2}} \mathbf{e}_{2}+X_{s_{3}} \mathbf{e}_{3}$

- $M_{t}$ in $D_{t}: \mathbf{X}_{t}=\mathbf{O M}_{t}=X_{t_{1}} \mathbf{e}_{1}+X_{t_{2}} \mathbf{e}_{2}+X_{t_{3}} \mathbf{e}_{3}$

The operators $\operatorname{grad}_{i}$ and $\operatorname{div}_{i}$ represent respectively the gradient and divergence in the configuration $D_{i}$. The transformations $\phi_{i j}$ describe the change from configuration $i$ to configuration $j$. For example, the transformation of the initial configuration to the rotating configuration is written as $\mathbf{X}_{r}=\phi_{0 r}\left(\mathbf{X}_{0}, t\right)$.

The Green tensors of the gradient of transformation are calculated using the configurations:

$$
\mathbf{F}_{0 r}=\frac{d \mathbf{X}_{r}}{d \mathbf{X}_{0}}, \quad \mathbf{F}_{r s}=\frac{d \mathbf{X}_{s}}{d \mathbf{X}_{r}}, \quad \mathbf{F}_{s t}=\frac{d \mathbf{X}_{t}}{d \mathbf{X}_{s}}, \quad \mathbf{F}_{0 s}=\frac{d \mathbf{X}_{s}}{d \mathbf{X}_{0}}
$$

One has relations between the different configurations, for instance: $\mathbf{F}_{0 s}=$ $\mathbf{F}_{r s} \cdot \mathbf{F}_{0 r}$. One can notice that the tensor $\mathbf{F}_{0 r}$ of a rigid transformation should satisfy: ${ }^{T} \mathbf{F}_{0 r} \cdot \mathbf{F}_{0 r}=\mathbf{F}_{0 r} \cdot{ }^{T} \mathbf{F}_{0 r}=\mathbf{I}$ with $\mathbf{I}$ the identity tensor.

\subsection{Equilibrium equations of the stationary state}

The ALE approach is taken to treat the stationary motion. The reference configuration of this step is $D_{r}$. We will write the balance equations on this configuration of reference. The conservation of quantities of movement on the stationary configuration $D_{s}$ leads to the equilibrium equations:

$$
\rho_{s} \gamma_{s}=\rho_{s} \mathbf{f}_{s}+\operatorname{div}_{s}\left(\boldsymbol{\sigma}_{s}\right)
$$

where $\rho_{s}$ is the density of the material in $D_{s}, \mathbf{f}_{s}$ the exterior body force applied in $D_{s}, \gamma_{s}$ the acceleration in $D_{s}$ and $\boldsymbol{\sigma}_{s}$ the Cauchy stress tensor in $D_{s}$. The equation (2) is written in the stationary configuration. One must describe all the terms of this equation in the reference configuration. 


\section{Density}

The mass conservation gives: $\rho_{s} V_{s}=\rho_{r} V_{r} \longrightarrow \rho_{s}=\frac{V_{r}}{V_{s}} \rho_{r}$ where $\rho_{r}$ is the density of the material in the rotating configuration $D_{r}$. One denotes $J=\frac{V_{s}}{V_{r}}$ the coefficient of volume expansion of the solid which equals $\operatorname{det}\left(\mathbf{F}_{r s}\right)$. One gets $\rho_{s}=\frac{\rho_{r}}{J}$.

\section{Acceleration}

The acceleration of a material point is calculated by Eulerian derivation versus time.

$$
\gamma_{s}=\frac{D^{2} \mathbf{X}_{s}}{D t^{2}}
$$

In the stationary state of the solid, the deformation of a material point in the current configuration depends only on its position in the rotating configuration. This deformation does not depend on time. We denote $\mathbf{X}_{s}=\phi_{r s}\left(\mathbf{X}_{r}\right)$. So the absolute velocity in the stationary configuration is calculated by:

$$
\mathbf{v}_{s}=\frac{D \mathbf{X}_{s}}{D t}=\frac{\partial \mathbf{X}_{s}}{\partial \mathbf{X}_{r}} \frac{\partial \mathbf{X}_{r}}{\partial t}
$$

The rigid movement is written as: $\mathbf{X}_{r}=\phi_{0 r}\left(\mathbf{X}_{0}, t\right)=\mathbf{C}(t)+\boldsymbol{\theta}(t)\left(\mathbf{X}_{0}-\mathbf{C}(0)\right)$ where $\mathbf{C}(t)$ and $\mathbf{C}(0)$ are respectively the position vectors of the rotation centre of the solid at time $t$ and at the initial time. $\boldsymbol{\theta}(t)$ is the orthogonal rotation matrix. So

$$
\begin{aligned}
\frac{\partial \mathbf{X}_{r}}{\partial t} & =\frac{\partial\left(\mathbf{C}(t)+\boldsymbol{\theta}(t)\left(\mathbf{X}_{0}-\mathbf{C}(0)\right)\right)}{\partial t} \\
& =\dot{\mathbf{C}}(t)+\dot{\boldsymbol{\theta}}(t)\left(\mathbf{X}_{0}-\mathbf{C}(0)\right) \\
& =\dot{\mathbf{C}}(t)+\dot{\boldsymbol{\theta}}(t) \boldsymbol{\theta}(t)^{-1}\left(\mathbf{X}_{r}-\mathbf{C}(t)\right)
\end{aligned}
$$

One defines:

$$
\boldsymbol{\Omega}\left(\mathbf{X}_{r}, t\right)=\dot{\mathbf{C}}(t)+\dot{\boldsymbol{\theta}}(t) \boldsymbol{\theta}(t)^{-1}\left(\mathbf{X}_{r}-\mathbf{C}(t)\right)
$$

the rotation vector of the solid. It is noted that the velocity of the material point of the reference configuration is the rotation vector. It is known if we know the speed of rotation. The expression (4) becomes:

$$
\mathbf{v}_{s}=\frac{D \mathbf{X}_{s}}{D t}=\mathbf{F}_{r s} \cdot \boldsymbol{\Omega}\left(\mathbf{X}_{r}, t\right)
$$

From (7), one computes the acceleration in (3):

$$
\begin{aligned}
\gamma_{s} & =\frac{D^{2} \mathbf{X}_{s}}{D t^{2}} \\
& =\frac{D \mathbf{v}_{s}}{D t} \\
& =\frac{D\left(\mathbf{F}_{r s} \cdot \boldsymbol{\Omega}\left(\mathbf{X}_{r}, t\right)\right)}{D t} \\
& =\frac{\partial\left(\mathbf{F}_{r s} \cdot \boldsymbol{\Omega}\left(\mathbf{X}_{r}, t\right)\right)}{\partial t}+\frac{\partial\left(\mathbf{F}_{r s} \cdot \boldsymbol{\Omega}\left(\mathbf{X}_{r}, t\right)\right)}{\partial \mathbf{X}_{r}} \cdot \boldsymbol{\Omega}\left(\mathbf{X}_{r}, t\right)
\end{aligned}
$$


So,

$$
\boldsymbol{\gamma}_{s}=\mathbf{F}_{r s} \cdot \frac{\partial\left(\boldsymbol{\Omega}\left(\mathbf{X}_{r}, t\right)\right)}{\partial t}+\operatorname{grad}_{r}\left(\mathbf{F}_{r s} \cdot \boldsymbol{\Omega}\left(\mathbf{X}_{r}, t\right)\right) \cdot \boldsymbol{\Omega}\left(\mathbf{X}_{r}, t\right)
$$

\section{Stress}

In equation (2), the stress tensor is the Cauchy tensor. This is the stress on the deformed configuration. It must be turned into the first or second Piola-Kirchhoff stress tensors which are stress fields of the reference configuration $[50,51]$.

Knowing the acceleration, the equation of equilibrium of the stationary state can be written in the rotating configuration as:

$$
\mathbf{F}_{r s} \cdot \frac{\partial\left(\boldsymbol{\Omega}\left(\mathbf{X}_{r}, t\right)\right)}{\partial t}+\operatorname{grad}_{r}\left(\mathbf{F}_{r s} \cdot \boldsymbol{\Omega}\left(\mathbf{X}_{r}, t\right)\right) \cdot \boldsymbol{\Omega}\left(\mathbf{X}_{r}, t\right)=\mathbf{f}_{s}+\frac{1}{\rho_{r}} \operatorname{div}_{r}\left(\mathbf{P}_{r s}\right)
$$

with the first Piola-Kirchhoff stress tensor such that

$$
\mathbf{P}_{r s}=\mathbf{F}_{r s} \mathbf{S}_{r}
$$

and the second Piola-Kirchhoff given by

$$
\mathbf{S}_{r}=J \mathbf{F}_{r s}^{-1} \boldsymbol{\sigma}_{s}^{T} \mathbf{F}_{r s}^{-1}
$$

Supposing that $\frac{\partial\left(\boldsymbol{\Omega}\left(\mathbf{X}_{r}, t\right)\right)}{\partial t}=0$ (without orthocentrifugal inertia force) the equation of equilibrium (10) is written as:

$$
\operatorname{grad}_{r}\left(\mathbf{F}_{r s} \cdot \boldsymbol{\Omega}\left(\mathbf{X}_{r}, t\right)\right) \cdot \boldsymbol{\Omega}\left(\mathbf{X}_{r}, t\right)=\mathbf{f}_{s}+\frac{1}{\rho_{r}} \operatorname{div}_{r}\left(\mathbf{F}_{r s} \mathbf{S}_{r}\right)
$$

\subsection{Equations of the vibrating state}

2.3.1. Balance equation of the vibrating state with respect to the stationary state

To describe the movement, the following notations are defined or recalled:

$$
\left\{\begin{array}{l}
\mathbf{X}_{r}=\phi_{0 r}\left(\mathbf{X}_{0}, t\right)=\mathbf{C}(t)+\boldsymbol{\theta}(t)\left(\mathbf{X}_{0}-\mathbf{C}(0)\right) \\
\mathbf{X}_{s}=\phi_{r s}\left(\mathbf{X}_{r}, t\right)=\mathbf{X}_{r}+\mathbf{U}_{s}\left(\mathbf{X}_{r}\right) \\
\mathbf{X}_{t}=\phi_{s t}\left(\mathbf{X}_{s}, t\right)=\mathbf{X}_{s}+\mathbf{u}\left(\mathbf{X}_{s}, t\right)=\mathbf{X}_{r}+\mathbf{U}_{s}\left(\mathbf{X}_{r}\right)+\mathbf{u}_{t}\left(\mathbf{X}_{r}, t\right)
\end{array}\right.
$$

with $\mathbf{U}_{s}\left(\mathbf{X}_{r}\right)$ representing the stationary displacement, $\mathbf{u}\left(\mathbf{X}_{s}, t\right)$ and $\mathbf{u}_{t}\left(\mathbf{X}_{r}, t\right)$ are small perturbations around the stationary state projected onto the stationary configuration and the configuration of reference respectively. The condition for an infinitesimal disturbance is written as:

$$
\operatorname{grad}_{s}\left(\mathbf{u}\left(\mathbf{X}_{s}, t\right)\right) \ll \mathbf{I}
$$

or

$$
\operatorname{grad}_{r}\left(\mathbf{u}_{t}\left(\mathbf{X}_{r}, t\right)\right) \ll \mathbf{I}
$$


In the vibrant configuration $D_{t}$, the equation of equilibrium is obtained in the same way as in the stationary case:

$$
\rho_{t} \gamma_{t}=\rho_{t} \mathbf{f}_{t}+\operatorname{div}_{t}\left(\boldsymbol{\sigma}_{t}\right)
$$

with $\rho_{t}$ representing the density of the material in $D_{t}, \mathbf{f}_{t}$ the exterior body force in $D_{t}, \gamma_{t}$ the acceleration in $D_{t}$ and $\boldsymbol{\sigma}_{t}$ the Cauchy stress tensor in $D_{t}$. Under the hypothesis of infinitesimal strains, the $D_{t}$ and $D_{s}$ configurations are confused. This implies that $\rho_{t}=\rho_{s}$. The velocity of a material point in the current configuration is calculated as:

$$
\frac{D \mathbf{X}_{t}}{D t}=\frac{D \phi_{s t}\left(\mathbf{X}_{s}, t\right)}{D t}=\frac{\partial \phi_{s t}\left(\mathbf{X}_{s}, t\right)}{\partial t}+\frac{\partial \phi_{s t}\left(\mathbf{X}_{s}, t\right)}{\partial \mathbf{X}_{s}} \frac{\partial \mathbf{X}_{s}}{\partial t}
$$

So,

$$
\frac{D \mathbf{X}_{t}}{D t}=\frac{\partial \phi_{s t}\left(\mathbf{X}_{s}, t\right)}{\partial t}+\mathbf{F}_{s t} \cdot \mathbf{v}_{s}\left(\mathbf{X}_{s}, t\right)
$$

The acceleration is

$$
\gamma_{t}=\frac{D^{2} \mathbf{X}_{t}}{D t^{2}}=\frac{D\left(\frac{\partial \phi_{s t}\left(\mathbf{X}_{s}, t\right)}{\partial t}+\mathbf{F}_{s t} \cdot \mathbf{v}_{s}\left(\mathbf{X}_{s}, t\right)\right)}{D t}
$$

After development, this leads to:

$$
\begin{aligned}
\gamma_{t}=\frac{\partial^{2}\left(\phi_{s t}\left(\mathbf{X}_{s}, t\right)\right)}{\partial t^{2}} & +2 \frac{\partial \mathbf{F}_{s t}}{\partial t} \cdot \mathbf{v}_{s}\left(\mathbf{X}_{s}, t\right) \\
& +\mathbf{F}_{s t} \cdot \frac{\partial \mathbf{v}_{s}\left(\mathbf{X}_{s}, t\right)}{\partial t}+\operatorname{grad}_{s}\left(\mathbf{F}_{s t} \cdot \mathbf{v}_{s}\left(\mathbf{X}_{s}, t\right)\right) \cdot \mathbf{v}_{s}\left(\mathbf{X}_{s}, t\right)
\end{aligned}
$$

The description of the movement allows to write $\mathbf{X}_{t}=\phi_{s t}\left(\mathbf{X}_{s}, t\right)=\mathbf{X}_{s}+$ $\mathbf{u}\left(\mathbf{X}_{s}, t\right)$ in the precedent formula and the expression (20) becomes:

$$
\begin{aligned}
\boldsymbol{\gamma}_{t}=\frac{\partial^{2}\left(\mathbf{u}\left(\mathbf{X}_{s}, t\right)\right)}{\partial t^{2}} & +2 \frac{\partial \mathbf{F}_{s t}}{\partial t} \cdot \mathbf{v}_{s}\left(\mathbf{X}_{s}, t\right) \\
+ & \mathbf{F}_{s t} \cdot \frac{\partial \mathbf{v}_{s}\left(\mathbf{X}_{s}, t\right)}{\partial t}+\operatorname{grad}_{s}\left(\mathbf{F}_{s t} \cdot \mathbf{v}_{s}\left(\mathbf{X}_{s}, t\right)\right) \cdot \mathbf{v}_{s}\left(\mathbf{X}_{s}, t\right)
\end{aligned}
$$

As in the stationary case, the increment of the second Piola-Kirchhoff tensor $\mathbf{S}_{t}$ is linked to the Cauchy stresses $\boldsymbol{\sigma}_{t}$ in the stationary configuration $D_{s}$ by:

$$
\mathbf{S}_{t}=J \mathbf{F}_{s t}^{-1} \boldsymbol{\sigma}_{t}^{T} \mathbf{F}_{s t}^{-1}
$$

The equilibrium equation is written as:

$$
\frac{\partial^{2} \mathbf{u}}{\partial t^{2}}+2 \frac{\partial \mathbf{F}_{s t}}{\partial t} \cdot \mathbf{v}_{s}+\mathbf{F}_{s t} \cdot \frac{\partial \mathbf{v}_{s}}{\partial t}+\operatorname{grad}_{s}\left(\mathbf{F}_{s t} \cdot \mathbf{v}_{s}\right) \cdot \mathbf{v}_{s}=\mathbf{f}_{t}+\frac{1}{\rho_{s}} \operatorname{div}_{s}\left(\mathbf{F}_{s t} \cdot \mathbf{S}_{t}\right)
$$


On the other hand, the acceleration of a material point is calculated as the derivative of velocity in the stationary configuration:

$$
\gamma_{s}=\frac{D\left(\mathbf{v}_{s}\left(\mathbf{X}_{s}, t\right)\right)}{D t}=\frac{\partial \mathbf{v}_{s}\left(\mathbf{X}_{s}, t\right)}{\partial t}+\operatorname{grad}_{s}\left(\mathbf{v}_{s}\left(\mathbf{X}_{s}, t\right)\right) \cdot \mathbf{v}_{s}\left(\mathbf{X}_{s}, t\right)
$$

The equation of equilibrium in the stationary configuration (2) can be written in the form:

$$
\frac{\partial \mathbf{v}_{s}\left(\mathbf{X}_{s}, t\right)}{\partial t}+\operatorname{grad}_{s}\left(\mathbf{v}_{s}\left(\mathbf{X}_{s}, t\right)\right) \cdot \mathbf{v}_{s}\left(\mathbf{X}_{s}, t\right)=\mathbf{f}_{s}+\frac{1}{\rho_{s}} \operatorname{div}_{s}\left(\boldsymbol{\sigma}_{s}\right)
$$

So by simple subtraction of the two equations (23) and (25), one obtains the vibrating balance equation around the stationary state:

$$
\begin{aligned}
\frac{\partial^{2} \mathbf{u}}{\partial t^{2}}+2 \frac{\partial \mathbf{F}_{s t}}{\partial t} \cdot \mathbf{v}_{s}+\left(\mathbf{F}_{s t}-\mathbf{I}\right) \frac{\partial \mathbf{v}_{s}}{\partial t} & +\operatorname{grad}_{s}\left(\left(\mathbf{F}_{s t}-\mathbf{I}\right) \cdot \mathbf{v}_{s}\right) \cdot \mathbf{v}_{s} \\
& =\left(\mathbf{f}_{t}-\mathbf{f}_{s}\right)+\frac{1}{\rho_{s}} \operatorname{div}_{s}\left(\mathbf{F}_{s t} \cdot \mathbf{S}_{t}-\boldsymbol{\sigma}_{s}\right)
\end{aligned}
$$

Recall that expression (1) combined with expressions (14) gives:

$$
\mathbf{F}_{s t}=\frac{d \mathbf{X}_{t}}{d \mathbf{X}_{s}}=\mathbf{I}+\operatorname{grad}_{s}\left(\mathbf{u}\left(\mathbf{X}_{s}, t\right)\right)
$$

By replacing it in the expression (26):

$$
\begin{aligned}
\frac{\partial^{2} \mathbf{u}}{\partial t^{2}}+2 \frac{\partial \operatorname{grad}_{s}(\mathbf{u})}{\partial t} \cdot \mathbf{v}_{s}+\operatorname{grad}_{s}(\mathbf{u}) & \frac{\partial \mathbf{v}_{s}}{\partial t}+\operatorname{grad}_{s}\left(\operatorname{grad}_{s}(\mathbf{u}) \cdot \mathbf{v}_{s}\right) \cdot \mathbf{v}_{s} \\
& =\left(\mathbf{f}_{t}-\mathbf{f}_{s}\right)+\frac{1}{\rho_{s}} \operatorname{div}_{s}\left(\mathbf{F}_{s t} \cdot \mathbf{S}_{t}-\boldsymbol{\sigma}_{s}\right)
\end{aligned}
$$

One defines $\tilde{\mathbf{S}}_{t}=\mathbf{S}_{t}-\boldsymbol{\sigma}_{s}$ which is the increment of the stress tensor which can be computed by the behaviour law of the solid material:

$$
\tilde{\mathbf{S}}_{t}=\mathbf{C}: \mathbf{e}
$$

with $\mathbf{C}$ is the tensor describing the behaviour of the material and $\mathbf{e}$ is the Green strain tensor

$$
\mathbf{e}=\frac{1}{2}\left(\operatorname{grad}_{s}(\mathbf{u})+\operatorname{grad}_{s}(\mathbf{u})^{T}+\operatorname{grad}_{s}(\mathbf{u})^{T} \operatorname{grad}_{s}(\mathbf{u})\right)
$$

Using the hypothesis of a small perturbation: $\left|\mathbf{F}_{s t}-\mathbf{I}\right| \ll 1$ or $\left|\operatorname{grad}_{s}(\mathbf{u})\right| \ll$ 1 , and neglecting all second order terms, the equation of equilibrium (27) can be written

$$
\begin{aligned}
\ddot{\mathbf{u}}+ & 2 \operatorname{grad}_{s}(\dot{\mathbf{u}}) \cdot \mathbf{v}_{s}+\operatorname{grad}_{s}(\mathbf{u}) \cdot \dot{\mathbf{v}}_{s}+\operatorname{grad}_{s}\left(\operatorname{grad}_{s}(\mathbf{u}) \cdot \mathbf{v}_{s}\right) \cdot \mathbf{v}_{s} \\
& =\left(\mathbf{f}_{t}-\mathbf{f}_{s}\right)+\frac{1}{\rho_{s}} \operatorname{div}_{s}\left(\tilde{\mathbf{S}}_{t}+\operatorname{grad}_{s}(\mathbf{u}) \cdot \boldsymbol{\sigma}_{s}\right)
\end{aligned}
$$

Physically, the terms in the formula for this balance equation are defined by: 
- The relative acceleration: $\ddot{\mathbf{u}}\left(\mathbf{X}_{s}, t\right)$

- The Coriolis acceleration: $2 \operatorname{grad}_{s}\left(\dot{\mathbf{u}}\left(\mathbf{X}_{s}, t\right)\right) \cdot \mathbf{v}_{s}$

- The ortho-centripetal acceleration: $\operatorname{grad}_{s}(\mathbf{u}) \cdot \dot{\mathbf{v}}_{s}$

- The centripetal acceleration: $\operatorname{grad}_{s}\left(\operatorname{grad}_{s}\left(\mathbf{u}\left(\mathbf{X}_{s}, t\right)\right) \cdot \mathbf{v}_{s}\right) \cdot \mathbf{v}_{s}$

- The body force: $\left(\mathbf{f}_{t}-\mathbf{f}_{s}\right)$

- The internal stress $\frac{1}{\rho_{s}} \operatorname{div}_{s}\left(\tilde{\mathbf{S}}_{t}+\operatorname{grad}_{s}(\mathbf{u}) \cdot \boldsymbol{\sigma}_{s}\right)$

\section{Remark:}

$\mathbf{v}_{s}$ is the rotational speed (because in the stationary state the displacement does not depend on time). So, in a state in which the rotational speed is constant, $\mathbf{v}_{s}$ is also a constant. The term of the Coriolis acceleration is proportional to $\mathbf{v}_{s}$ and that of centripetal acceleration is proportional to the square of that speed. In this case, the ortho-centripetal acceleration is zero.

2.3.2. Balance equation of the vibrating state with respect to the rotating state

The position of the material point in the vibrant configuration is written as (14):

$$
\mathbf{X}_{t}=\mathbf{X}_{r}+\mathbf{U}_{s}\left(\mathbf{X}_{r}\right)+\mathbf{u}_{t}\left(\mathbf{X}_{r}, t\right)
$$

As in the development of (18) to (20), the term of the acceleration of a material point with respect to the rotating configuration is given by:

$$
\begin{aligned}
\boldsymbol{\gamma}_{t}=\frac{\partial^{2}\left(\mathbf{u}_{t}\left(\mathbf{X}_{R}, t\right)\right)}{\partial t^{2}}+2 \frac{\partial \mathbf{F}_{r t}}{\partial t} \cdot \boldsymbol{\Omega}\left(\mathbf{X}_{R}, t\right) \\
\quad+\mathbf{F}_{r t} \cdot \frac{\partial \boldsymbol{\Omega}\left(\mathbf{X}_{R}, t\right)}{\partial t}+\operatorname{grad}_{r}\left(\mathbf{F}_{r t} \cdot \boldsymbol{\Omega}\left(\mathbf{X}_{R}, t\right)\right) \cdot \boldsymbol{\Omega}\left(\mathbf{X}_{R}, t\right)
\end{aligned}
$$

with $\boldsymbol{\Omega}\left(\mathbf{X}_{R}, t\right)$ is the rotation vector of the rigid motion.

Therefore, the equilibrium equations with respect to the rotating configuration are written:

$$
\frac{\partial^{2} \mathbf{u}_{t}}{\partial t^{2}}+2 \frac{\partial \mathbf{F}_{r t}}{\partial t} \cdot \boldsymbol{\Omega}+\mathbf{F}_{r t} \cdot \frac{\partial \boldsymbol{\Omega}}{\partial t}+\operatorname{grad}_{r}\left(\mathbf{F}_{r t} \cdot \boldsymbol{\Omega}\right) \cdot \boldsymbol{\Omega}=\mathbf{f}_{t}+\frac{1}{\rho_{r}} \operatorname{div}_{r}\left(\mathbf{F}_{r t} \cdot \mathbf{S}_{r}\right)
$$

with $\mathbf{S}_{r}$ representing the second Piola-Kirchhoff stress tensor.

\section{Geometrically nonlinear circular ring model}

\subsection{Description of the model}

The description of the circular ring model representing a tire is illustrated in figure (2). The tread is described as a circular beam. The sides are 


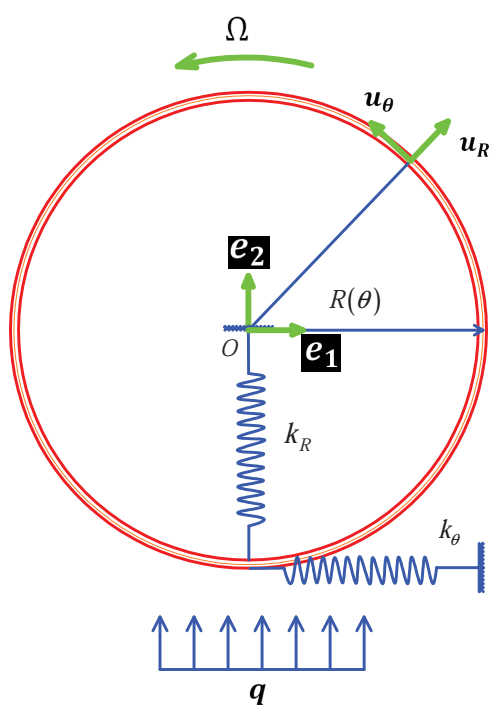

Figure 2: Description of the circular ring model

modelled using radial and tangential springs. The pressure is modelled as a uniform load on the ring. Finally, the tire/road contact is described by a force field applied to the base of the ring. The radial and tangential springs are fixed at the other end.

The circular ring has a radius $R$, a straight section $A$ and a thickness $e$. The beam is assumed very thin with $\frac{e}{R} \ll 1$ which is a condition on the geometry of the tread. It behaves like a beam which must satisfy the following assumptions:

- The radius of curvature of the medium line and its length are large relatively to the largest transverse dimension of the cross section.

- The area of the cross section does not change after deformation.

- The beam is of Cosserat-Timoshenko type. This means that the shear effect is taken into account and that there is no warping of the cross section.

- The material of the beam is linear elastic.

$k_{R}, k_{\theta}$ are respectively the radial and tangential stiffnesses of the springs.

\subsection{Stationary motion}

\subsubsection{Description of the motion}

Each material point in the rotating configuration is defined by two variables $(z, \theta)$ in the polar coordinates defined by $\left(\mathbf{u}_{R}, \mathbf{u}_{\theta}\right)$ with $z$ varying in 
the range $\left[-\frac{e}{2} ; \frac{e}{2}\right]$ and $\theta$ in $[0 ; 2 \pi]$.

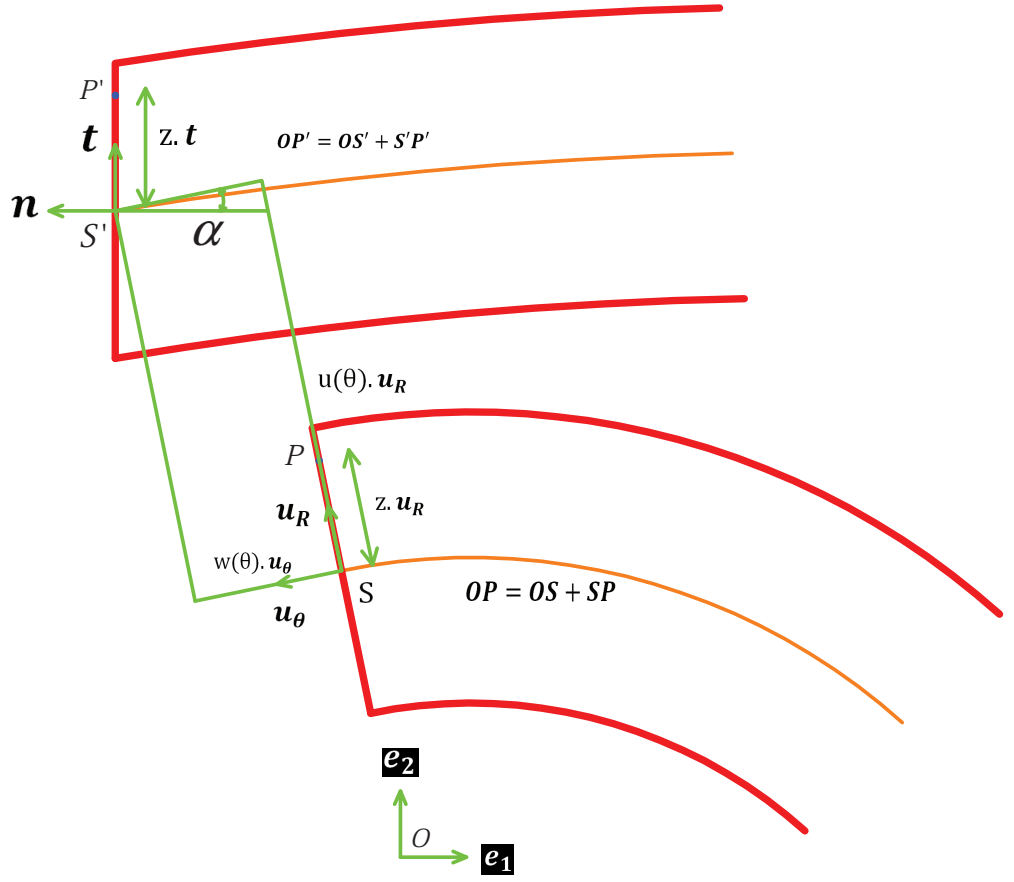

Figure 3: Description of the transformation

In the rotating configuration, a material point can be represented in the following way:

$$
\mathbf{O P}=\mathbf{O S}+\mathbf{S P}=(R+z) \mathbf{u}_{R}
$$

with $S$ is a point on the neutral fibre of the beam. $S$ and $P$ belong to the same section. By switching to the stationary configuration, a displacement field is applied so that $P \rightarrow P^{\prime}$ and $S \rightarrow S^{\prime}$. Point $S$ moves to point $S^{\prime}$ by two translations $(u(\theta), w(\theta))$. Point $P$ turns by an angle $\alpha$ to point $P^{\prime}$, see figure 3 . Therefore, the transformation of the material vector is defined by:

$$
\mathbf{O P}^{\prime}=\mathbf{O S}^{\prime}+\mathbf{S}^{\prime} \mathbf{P}^{\prime}=(R+u+z \cos \alpha) \mathbf{u}_{R}+(w+z \sin \alpha) \mathbf{u}_{\theta}
$$

The displacement vector of a material point is then:

$$
\mathbf{u}=\mathbf{O} \mathbf{P}^{\prime}-\mathbf{O P}=(u+z(\cos \alpha-1)) \mathbf{u}_{R}+(w+z \sin \alpha) \mathbf{u}_{\theta}
$$

One also has the transformation matrix:

$$
\left(\begin{array}{l}
\mathbf{t} \\
\mathbf{n}
\end{array}\right)=\left(\begin{array}{cc}
\cos \alpha & \sin \alpha \\
-\sin \alpha & \cos \alpha
\end{array}\right)\left(\begin{array}{l}
\mathbf{u}_{R} \\
\mathbf{u}_{\theta}
\end{array}\right)
$$




\subsubsection{Strain tensor}

The tensor of the gradient of the transformation is calculated by:

$$
\mathbf{F}=\mathbf{I}+\operatorname{grad}(\mathbf{u})
$$

and the strain tensor of the transformation is computed by:

$$
\mathbf{e}=\frac{1}{2}\left({ }^{T} \mathbf{F} \cdot \mathbf{F}-\mathbf{I}\right)
$$

The detailed computation is given in Appendix A and leads to the formulas for each component:

$$
\begin{aligned}
e_{R R}= & 0 \\
e_{R \theta}= & e_{\theta R}=\frac{1}{2 R}\left(\zeta^{2}-\zeta+1\right)\left(\left(u^{\prime}-w\right) \cos \alpha+\left(R+u+w^{\prime}\right) \sin \alpha\right) \\
e_{\theta \theta}= & \frac{1-2 \zeta+3 \zeta^{2}}{2 R^{2}}\left(\left(u^{\prime}-w\right)^{2}+\left(R+u+w^{\prime}\right)^{2}\right. \\
& +2 R \zeta\left(\alpha^{\prime}+1\right)\left(\left(R+u+w^{\prime}\right) \cos \alpha-\left(u^{\prime}-w\right) \sin \alpha\right) \\
& \left.+R^{2} \zeta^{2}\left(\alpha^{\prime}+1\right)^{2}\right)-\frac{1}{2}
\end{aligned}
$$

\subsubsection{Behaviour law and internal forces}

Material behaviour of the elastic ring is assumed linear elastic. The stress tensor can be written with the engineering notations as:

$$
\left(\begin{array}{c}
S_{\theta \theta} \\
S_{R \theta}
\end{array}\right)=\left(\begin{array}{cc}
E & 0 \\
0 & G
\end{array}\right)\left(\begin{array}{c}
e_{\theta \theta} \\
2 e_{R \theta}
\end{array}\right)
$$

with $E$ and $G$ are the Young and shear modulus. Active forces on the cross section are the normal force $N$, the shear force $V$ and the moment $M$, which are calculated as following in the reference configuration $\left(\mathbf{u}_{R}, \mathbf{u}_{\theta}\right)$ :

$$
\left\{\begin{array}{l}
N=\int_{A} S_{\theta \theta} d A \\
V=\int_{A} S_{R \theta} d A \\
M=\int_{A} z S_{\theta \theta} d A
\end{array}\right.
$$

with $A$ is the cross-section area. The detailed expressions of these quantities are given in Appendix B.

One denotes the force on the cross section in the reference configuration as:

$$
\mathbf{f}_{r}=V \mathbf{u}_{R}+N \mathbf{u}_{\theta}
$$


This force is written in the stationary configuration using (36):

$$
\mathbf{f}=\left(\begin{array}{cc}
\cos \alpha & \sin \alpha \\
-\sin \alpha & \cos \alpha
\end{array}\right) \mathbf{f}_{r}=N \mathbf{n}+V \mathbf{t}
$$

\section{Remark:}

The components of the force on the cross-section relative to the reference configuration $\mathbf{f}_{r}$ equal those of the force on the cross-section in the stationary configuration $\mathbf{f}$ (cf. Simo [44]).

\subsubsection{Equilibrium equations}

The equilibrium equations of the stationary state relatively to the rotating configuration, are proved in the precedent section (cf. 2.2) in equation (13). To simplify the writing of formulas, the index " $r$ " is deleted from all operators. From that moment, all calculations are in the rotating configuration (the non-deformed configuration). The equilibrium equations yield:

$$
\rho \operatorname{grad}(\mathbf{F} . \boldsymbol{\Omega}(\mathbf{X}, t)) . \boldsymbol{\Omega}(\mathbf{X}, t)=\rho \mathbf{f}+\operatorname{div}(\mathbf{F} . \mathbf{S})
$$

with $\rho$ is the density, $\mathbf{F}$ the tensor of the gradient of transformation, $\rho \mathbf{f}=\mathbf{q}^{v}$ the body force applied on the ring, $\boldsymbol{\Omega}$ the rotation vector, $\mathbf{S}$ the second PiolaKirchhoff stress tensor. F.S $=\mathbf{P}$ is the first Piola-Kirchhoff stress tensor. To set the equilibrium equations on the ring, these equations are integrated on a beam portion.

\section{Equilibrium of forces}

Formula (43) is integrated on a volume $\mathfrak{D}$ of reference length " $d s$ " along the neutral axis in the deformed configuration:

$$
\int_{\mathcal{D}} \rho \operatorname{grad}(\mathbf{F} \cdot \boldsymbol{\Omega}(\mathbf{X}, t)) \cdot \boldsymbol{\Omega}(\mathbf{X}, t) d V=\int_{\mathcal{D}} \mathbf{q}^{v} d V+\int_{\mathcal{D}} \operatorname{div}(\mathbf{P}) d V
$$

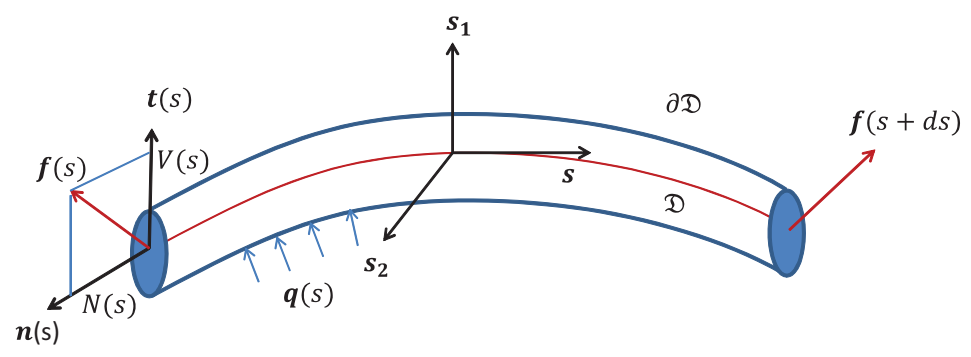

Figure 4: Equilibrium around a portion of the beam. 
The tensor $\mathbf{P}$ is written under the form $\mathbf{P}=\mathbf{P}_{1} \otimes \mathbf{s}_{1}+\mathbf{P}_{2} \otimes \mathbf{s}_{2}+\mathbf{P}_{3} \otimes \mathbf{s}$ where $\left(\mathbf{s}_{1}, \mathbf{s}_{2}, \mathbf{s}\right)$ are the unit vectors of the orthogonal curvilinear reference system. $\mathbf{s}$ is the vector tangential to the neutral axis. One recall:

$$
\mathbf{f}=\int_{A} \mathbf{P} \cdot \mathbf{s} d A
$$

the active force on the cross section in the deformed configuration. The projection of $\mathbf{f}$ on two unit vectors in a basis of the current cross-section $(\mathbf{t}, \mathbf{n})$ gives respectively the shear and normal forces $(V, N)$ (cf. relation 42). The theorem of Green-Ostrogradski allows writing:

$$
\int_{\mathcal{D}} \operatorname{div}(\mathbf{P}) d V=\mathbf{f}(s+d s)-\mathbf{f}(s)+\mathbf{q}(s) d s
$$

$\mathbf{q}=\int_{\partial A} \mathbf{q}^{\mathbf{s}} d l$ are the surface forces on the beam from the air pressure, the contact forces and the forces of the springs. $l$ is the curvilinear abscissa of the edge of the cross section $\partial A$.

Replacing the relation (46) in equation (44) yields:

$$
\int_{\mathcal{D}} \rho \operatorname{grad}(\mathbf{F} \cdot \boldsymbol{\Omega}(\mathbf{X}, t)) \cdot \boldsymbol{\Omega}(\mathbf{X}, t) d V=\mathbf{f}(s+d s)-\mathbf{f}(s)+\mathbf{q}(s) d s+\int_{\mathcal{D}} \mathbf{q}^{v} d V
$$

$d s$ is small enough to have $(\rho \operatorname{grad}(\mathbf{F} . \boldsymbol{\Omega}(\mathbf{x}, t)) . \boldsymbol{\Omega}(\mathbf{x}, t))$ and $\mathbf{q}^{v}$ constant along $d s$. Equation (47) becomes:

$d s \int_{A} \rho \operatorname{grad}(\mathbf{F} \cdot \boldsymbol{\Omega}(\mathbf{X}, t)) \cdot \boldsymbol{\Omega}(\mathbf{X}, t) d A=\mathbf{f}(s+d s)-\mathbf{f}(s)+\mathbf{q}(s) d s+d s \int_{A} \mathbf{q}^{v} d A$

The equilibrium of forces is thus written as:

$$
\frac{\partial \mathbf{f}}{\partial s}+\oint_{\partial A} \mathbf{q}^{s} d l+\int_{A} \mathbf{q}^{v} \cdot d A=\int_{A} \rho \operatorname{grad}(\mathbf{F} \cdot \boldsymbol{\Omega}(\mathbf{X}, t)) \cdot \boldsymbol{\Omega}(\mathbf{X}, t) d A
$$

Equation (49) represents the equilibrium of forces on a piece of beam. $\frac{\partial \mathbf{f}}{\partial s}$ is a variation in the forces on two straight sections between positions $s$ and $s+d s . \int_{\partial A} \mathbf{q}^{s} d l$ is the sum of surface forces on the beam. $\int_{A} \mathbf{q}^{v} \cdot d A$ is the sum of the body forces. $\int_{A} \rho \operatorname{grad}(\mathbf{F} \cdot \boldsymbol{\Omega}(\mathbf{X}, t)) \cdot \boldsymbol{\Omega}(\mathbf{X}, t) d A$ is the centripetal force linked to the rotation.

\section{Equilibrium of the moment}

In the same way, the equation of balance of the moment is built. From the equation (43), a cross product is applied to the left. Then the expression 
is integrated on the domain $\mathfrak{D}$ :

$$
\begin{aligned}
\int_{\mathfrak{D}} \mathbf{S}^{\prime} \mathbf{P}^{\prime} \wedge(\rho \operatorname{grad}(\mathbf{F} \cdot \boldsymbol{\Omega}(\mathbf{X}, t)) \cdot \boldsymbol{\Omega}(\mathbf{X}, t)) d V \\
=\int_{\mathfrak{D}} \mathbf{S}^{\prime} \mathbf{P}^{\prime} \wedge \mathbf{q}^{v} d V+\int_{\mathfrak{D}} \mathbf{S}^{\prime} \mathbf{P}^{\prime} \wedge \operatorname{div}(\mathbf{P}) d V
\end{aligned}
$$

Supposing $d s$ small enough:

$$
\begin{aligned}
\int_{\mathcal{D}} & \mathbf{S}^{\prime} \mathbf{P}^{\prime} \wedge(\rho \operatorname{grad}(\mathbf{F} \cdot \boldsymbol{\Omega}(\mathbf{X}, t)) \cdot \boldsymbol{\Omega}(\mathbf{X}, t)) d V \\
& =d s \int_{A} \mathbf{S}^{\prime} \mathbf{P}^{\prime} \wedge(\rho \operatorname{grad}(\mathbf{F} \cdot \boldsymbol{\Omega}(\mathbf{X}, t)) \cdot \boldsymbol{\Omega}(\mathbf{X}, t)) d A \\
\int_{\mathcal{D}} \mathbf{S}^{\prime} \mathbf{P}^{\prime} \wedge \mathbf{q}^{v} d V & =d s \int_{A} \mathbf{S}^{\prime} \mathbf{P}^{\prime} \wedge \mathbf{q}^{v} d A
\end{aligned}
$$

With the developments of Appendix C, the equation of the equilibrium of the moment becomes:

$$
\begin{aligned}
& \frac{\partial \mathbf{M}}{\partial s}+\frac{\partial \mathbf{O} \mathbf{S}^{\prime}}{\partial s} \wedge \mathbf{f}+\int_{A} \mathbf{S}^{\prime} \mathbf{P}^{\prime} \wedge \mathbf{q}^{v} d A+\int_{\partial A}\left(\mathbf{S}^{\prime} \mathbf{P}^{\prime} \wedge \mathbf{q}^{s}\right) d l \\
& -\int_{A} \rho \mathbf{S}^{\prime} \mathbf{P}^{\prime} \wedge \operatorname{grad}(\mathbf{F} \cdot \boldsymbol{\Omega}(\mathbf{X}, t)) \cdot \boldsymbol{\Omega}(\mathbf{X}, t) d A=0
\end{aligned}
$$

The equations (49) and (52) allow finding the equations of equilibrium of beams set by Davi [47], Simo [44], Antman [35] under the following form in the stationary configuration $(\mathbf{t}, \mathbf{n})$ :

$$
\left\{\begin{array}{l}
\frac{\partial \mathbf{f}}{\partial s}+\mathbf{q}=0 \\
\frac{\partial \mathbf{M}}{\partial s} \cdot \mathbf{s}+\left(\frac{\partial \mathbf{O S}^{\prime}}{\partial s} \wedge \mathbf{f}\right) \cdot \mathbf{s}+g=0
\end{array}\right.
$$

with:

$\mathbf{f}=\left(\begin{array}{c}V \\ N\end{array}\right)$ the active forces on the cross section in the configuration $(\mathbf{n}, \mathbf{t})$ (cf. relation 42).

$\mathbf{M}$ is the moment in the cross-section.

$\mathbf{q}=\int_{\partial A} \mathbf{q}^{\mathbf{s}} d l+\int_{A} \mathbf{q}^{v} d A-\int_{A} \rho \operatorname{grad}(\mathbf{F} \cdot \boldsymbol{\Omega}(\mathbf{X}, t)) \cdot \boldsymbol{\Omega}(\mathbf{X}, t) d A$ are the applied forces. 


$$
\begin{aligned}
g= & \left(\int_{\partial A} \mathbf{S}^{\prime} \mathbf{P}^{\prime} \wedge \mathbf{q}^{s} d l+\int_{A} \mathbf{S}^{\prime} \mathbf{P}^{\prime} \wedge \mathbf{q}^{v} d A\right. \\
& \left.-\int_{A} \rho \mathbf{S}^{\prime} \mathbf{P}^{\prime} \wedge \operatorname{grad}(\mathbf{F} \cdot \mathbf{\Omega}(\mathbf{X}, t)) \boldsymbol{\Omega}(\mathbf{X}, t) d A\right) \cdot \mathbf{S}
\end{aligned}
$$

is the applied moment along the beam. The equations of equilibrium (53) are in the local basis of the deformed configuration $(\mathbf{t}, \mathbf{n})$. They can be written in the reference configuration $\left(\mathbf{u}_{R}, \mathbf{u}_{\theta}\right)$ :

$$
\left\{\begin{array}{l}
\frac{\partial}{\partial s}\left(\left(\begin{array}{cc}
\cos \alpha & -\sin \alpha \\
\sin \alpha & \cos \alpha
\end{array}\right)\left(\begin{array}{c}
V \\
N
\end{array}\right)\right)+\mathbf{q}=0 \\
\frac{\partial \mathbf{M}}{\partial s} \cdot \mathbf{s}+\left(\frac{\partial \mathbf{O S}^{\prime}}{\partial s} \wedge \mathbf{f}\right) \cdot \mathbf{s}+g=0
\end{array}\right.
$$

One must calculate the applied forces and moments on the beam in the reference configuration $\left(\mathbf{u}_{R}, \mathbf{u}_{\theta}\right)$.

\subsubsection{Computation of surface forces}

In our case, these forces consist of the air pressure, the contact force and the reactions of springs.

\section{- Air pressure}

Air pressure results in a pressure $p$ uniform along the ring. It is in the direction of $\mathbf{u}_{R}$ in the case of linear deformation. For the nonlinear case, the pressure follows the deformation. Therefore it is expressed as:

$$
\mathbf{p}=-p \mathbf{O} \mathbf{z} \wedge\left(\frac{\partial \mathbf{O S}^{\prime}}{\partial s}\right)=-p \mathbf{O} \mathbf{z} \wedge\left(\frac{\partial \mathbf{O S} \mathbf{S}^{\prime}}{R \partial \theta}\right)
$$

\section{- The contact}

At first, the tire/road contact is simply modelled by a point force in the radial direction $\left(\begin{array}{c}f_{R} \\ 0\end{array}\right)$.

\section{- The springs}

The forces are given by $\left(\begin{array}{c}f_{R}^{\text {spring }}=k_{R} \cdot \Delta l_{R} \\ f_{\theta}^{\text {spring }}=k_{\theta} \cdot \Delta l_{\theta}\end{array}\right)$. The elongations $\left(\begin{array}{c}\Delta l_{R} \\ \Delta l_{\theta}\end{array}\right)$ are computed by:

$$
\left(\begin{array}{c}
\Delta l_{R}=\sqrt{\left(l_{0}+u\right)^{2}+w^{2}}-l_{0} \\
\Delta l_{\theta}=\sqrt{\left(l_{0}+w\right)^{2}+u^{2}}-l_{0}
\end{array}\right) \cong\left(\begin{array}{c}
u \\
w
\end{array}\right)
$$


assuming that the initial lengths of the springs $l_{0}$ are very large compared to their displacements $u \ll l_{0}$ et $w \ll l_{0}$. The components $\left(f_{R}^{\text {spring }}, f_{\theta}^{\text {spring }}\right)$ are respectively in the direction of $\mathbf{u}_{R}$ and $\mathbf{u}_{\theta}$ in the case of linear deformation. For the nonlinear case, these components follow the deformation of the neutral axis. To sum up, the surface forces are in the reference system $\left(\mathbf{u}_{R}, \mathbf{u}_{\theta}\right)$ :

$$
\int_{\partial A} \mathbf{q}^{s} d l=\mathbf{p}+\left(\begin{array}{c}
f_{R}-f_{R}^{\text {spring }} \\
-f_{\theta}^{\text {spring }}
\end{array}\right)
$$

\subsubsection{Calculation of body forces}

In this work, the effect of the weight of the material is neglected. There remains only the inertial force bound to the rotation. From the equation of equilibrium (53), the centripetal force is calculated by:

$$
\int_{A} \rho \operatorname{grad}(\mathbf{F} \cdot \boldsymbol{\Omega}(\mathbf{X}, t)) \cdot \boldsymbol{\Omega}(\mathbf{X}, t) d A
$$

with $\boldsymbol{\Omega}(\mathbf{X}, t)=\dot{\mathbf{C}}(t)+\dot{\boldsymbol{\theta}}(t) \cdot \boldsymbol{\theta}(t)^{-1} \cdot[\mathbf{X}-\mathbf{C}(t)]$ the rotation vector (see equation (6)). We suppose that $\dot{\mathbf{C}}(t)=\mathbf{C}(t)=\mathbf{C}(0)=\mathbf{0}$ meaning that the centre of the wheel is fixed and is the origin of the reference system.

$\boldsymbol{\theta}(t)=\left(\begin{array}{cc}\cos \theta & -\sin \theta \\ \sin \theta & \cos \theta\end{array}\right)$ is the rotation matrix. So, with $\mathbf{X}=(R+z) \mathbf{u}_{R}$ and $\Omega=\dot{\theta}$, we get:

$$
\boldsymbol{\Omega}(\mathbf{X}, t)=\Omega(R+z) \mathbf{u}_{\theta}
$$

The computation developed in Appendix D yields the body force given by integrating over the cross section in the reference system $\left(\mathbf{u}_{R}, \mathbf{u}_{\theta}\right)$ :

$$
-\rho \int_{A} \operatorname{grad}(\mathbf{F} \boldsymbol{\Omega}(\mathbf{X}, t)) \cdot \boldsymbol{\Omega}(\mathbf{X}, t) d A=-\rho \Omega^{2} A\left(\begin{array}{c}
-R+u^{\prime \prime}-2 w^{\prime}-u \\
w^{\prime \prime}+2 u^{\prime}-w
\end{array}\right)
$$

\subsubsection{Calculation of the moment applied to the beam}

The surface forces move and revolve around a node while the beam is deformed. Therefore, they do not create moment on the beam because all quantities are independent of the variable $z$ (see relation (57)):

$$
\int_{\partial A} z \cdot \mathbf{t} \wedge \mathbf{q}^{s} d l=0
$$

Moreover, the weight of the beam is neglected. So, the moment is created by the centripetal body force. Using (D.5), the moment can be calculated 
by:

$$
g=\left(-\rho \int_{A} z \mathbf{t} \wedge \operatorname{grad}(\mathbf{F} . \boldsymbol{\Omega}(\mathbf{x}, t)) \cdot \boldsymbol{\Omega}(\mathbf{x}, t) d A\right) \cdot \mathbf{s}=-\rho \Omega^{2} I \alpha^{\prime \prime}
$$

In conclusion, the combination of equations (55), (59) and (60) provides the equations of equilibrium in the basis of the configuration of reference $\left(\mathbf{u}_{R}, \mathbf{u}_{\theta}\right)$ :

$$
\left\{\begin{array}{c}
\frac{1}{R} \frac{d}{d \theta}\left(\left(\begin{array}{cc}
\cos \alpha & -\sin \alpha \\
\sin \alpha & \cos \alpha
\end{array}\right)\left(\begin{array}{c}
V \\
N
\end{array}\right)\right)+\mathbf{p}-\left(\begin{array}{c}
f_{R}^{\text {spring }}-f_{R} \\
f_{\theta}^{\text {spring }}
\end{array}\right) \\
-\rho \Omega^{2} A\left(\begin{array}{c}
-R+u^{\prime \prime}-2 w^{\prime}-u \\
w^{\prime \prime}+2 u^{\prime}-w
\end{array}\right)=0 \\
\frac{1}{R} \frac{d M}{d \theta}+\frac{1}{R}\left(\frac{\partial O S^{\prime}}{\partial \theta} \wedge\left(\begin{array}{cc}
\cos \alpha & -\sin \alpha \\
\sin \alpha & \cos \alpha
\end{array}\right)\left(\begin{array}{c}
V \\
N
\end{array}\right)\right) . \mathbf{s}-\rho \Omega^{2} I \alpha^{\prime \prime}=0
\end{array}\right.
$$

with $M, N, V$ are given by (B.2).

\subsection{Dynamic motion}

\subsubsection{Different model configurations}

Configurations of the system are summarized in table (1). By switching

\begin{tabular}{|c|c|c|c|}
\hline Initial & Rotating & Stationary & Vibrating \\
\hline \multirow{2}{*}{$\mathbf{X}_{0}=R \cdot \mathbf{u}_{R}$} & $\left\{\begin{array}{l}\mathbf{X}_{r}=\boldsymbol{\theta} R \cdot \mathbf{u}_{R} \\
\boldsymbol{\Omega}\left(\mathbf{X}_{r}\right)=\dot{\theta} R \mathbf{u}_{\theta}\end{array}\right.$ & $\left\{\begin{array}{l}\mathbf{X}_{s}=\mathbf{X}_{r}+\mathbf{U} \\
\mathbf{U}=(u, w, \alpha)\end{array}\right.$ & $\left\{\begin{array}{l}\mathbf{X}_{t}=\mathbf{X}_{s}+\mathbf{U}_{t} \\
\mathbf{U}_{t}=\left(u_{t}, w_{t}, \alpha_{t}\right)\end{array}\right.$ \\
\hline
\end{tabular}

Table 1: Different configurations

to the vibrating configuration, a new displacement field must be defined:

$$
\left\{\begin{array}{l}
\mathrm{OS}^{\prime} \rightarrow \mathrm{OS}^{\prime \prime} \\
\mathbf{S}^{\prime} \mathbf{P}^{\prime} \rightarrow \mathbf{S}^{\prime \prime} \mathbf{P}^{\prime \prime}
\end{array}\right.
$$

The overall transformation of the rotating configuration to the vibrant configuration leads to:

$$
\left\{\begin{array}{l}
\mathrm{OS} \rightarrow \mathrm{OS}^{\prime \prime} \\
\mathrm{SP} \rightarrow \mathrm{S}^{\prime \prime} \mathbf{P}^{\prime \prime}
\end{array}\right.
$$

The total displacement is written as:

$$
\mathbf{u}=\mathbf{u}_{s}+\mathbf{u}_{t}
$$

with $\mathbf{u}_{s}$ is the stationary displacement and $\mathbf{u}_{t}$ is the dynamic perturbation. 


\subsubsection{Movement relative to the rotating configuration}

In this section, all developments are performed in the local basis $\left(\mathbf{u}_{R}, \mathbf{u}_{\theta}\right)$ of the rotating configuration. The total and stationary displacements are given by:

$$
\begin{gathered}
\mathbf{u}=\left(u+u_{t}+z\left(\cos \left(\alpha+\alpha_{t}\right)-1\right)\right) \mathbf{u}_{R}+\left(w+w_{t}+z \sin \left(\alpha+\alpha_{t}\right)\right) \mathbf{u}_{\theta} \\
\mathbf{u}_{s}=(u+z(\cos \alpha-1)) \mathbf{u}_{R}+(w+z \sin \alpha) \mathbf{u}_{\theta}
\end{gathered}
$$

The developments from the section on the stationary motion can be used. In particular, the gradient of the Green Lagrange transformation (A.3) relative to the rotating configuration is given by:

$$
\mathbf{F}_{r t}=\mathbf{I}+\operatorname{grad}_{r}(\mathbf{u})=\mathbf{I}+\operatorname{grad}_{r}\left(\mathbf{u}_{s}+\mathbf{u}_{t}\right)
$$

with:

$$
\begin{aligned}
& \mathbf{F}_{r t}= \\
& \left(\begin{array}{cc}
\cos \left(\alpha+\alpha_{t}\right) & \frac{1}{R+z}\left(u^{\prime}+u_{t}^{\prime}-w-w_{t}-z \sin \left(\alpha+\alpha_{t}\right)\left(1+\alpha^{\prime}+\alpha_{t}^{\prime}\right)\right) \\
\sin \left(\alpha+\alpha_{t}\right) & \frac{1}{R+z}\left(R+u+u_{t}+w^{\prime}+w_{t}^{\prime}+z \cos \left(\alpha+\alpha_{t}\right)\left(1+\alpha^{\prime}+\alpha_{t}^{\prime}\right)\right)
\end{array}\right)
\end{aligned}
$$

Neglecting the second order terms in $\mathbf{u}_{t}$ yields:

$$
\mathbf{F}_{r t}=\left(\begin{array}{cc}
\cos \alpha-\alpha_{t} \sin \alpha & \frac{1}{R+z}\left(\begin{array}{c}
u^{\prime}+u_{t}^{\prime}-w-w_{t}-z \alpha_{t}^{\prime} \sin \alpha \\
-z\left(\sin \alpha+\alpha_{t} \cos \alpha\right)\left(1+\alpha^{\prime}\right)
\end{array}\right) \\
\sin \alpha+\alpha_{t} \cos \alpha & \frac{1}{R+z}\left(\begin{array}{c}
R+u+u_{t}+w^{\prime}+w_{t}^{\prime}+z \alpha_{t}^{\prime} \cos \alpha \\
+z\left(\cos \alpha-\alpha_{t} \sin \alpha\right)\left(1+\alpha^{\prime}\right)
\end{array}\right)
\end{array}\right)
$$

\subsubsection{Analytical equilibrium equations in the rotating configuration}

As previously the developments made to establish the stationary equations are used again for the dynamic equations relatively to the rotating configuration. The hypothesis of small perturbations gives:

$$
\frac{u_{t}}{R} \ll 1, \quad \frac{w_{t}}{R} \ll 1, \quad \alpha_{t} \ll 1
$$


We first consider the case without rotation. The balance equations of the stationary case are taken from (61) by deleting the terms of rotation:

$$
\begin{aligned}
& \frac{1}{R}\left(V^{\prime} \cos \alpha-N^{\prime} \sin \alpha-\left(\alpha^{\prime}+1\right)(V \sin \alpha+N \cos \alpha)\right) \\
& +p \frac{R+u+w^{\prime}}{R}+f_{R}-k_{R} u=0 \\
& \frac{1}{R}\left(V^{\prime} \sin \alpha+N^{\prime} \cos \alpha+\left(\alpha^{\prime}+1\right)(V \cos \alpha-N \sin \alpha)\right)-p \frac{u^{\prime}-w}{R}-k_{\theta} w=0 \\
& \frac{1}{R} M^{\prime}+\frac{1}{R}\left(\left(u^{\prime}-w\right)(V \sin \alpha+N \cos \alpha)\right. \\
& \left.-\left(R+u+w^{\prime}\right)(V \cos \alpha-N \sin \alpha)\right)=0
\end{aligned}
$$

with $N, V$ and $M$ given by relation (B.2). By replacing $(u, w, \alpha)$ in these equations by $\left(u+u_{t}, w+w_{t}, \alpha+\alpha_{t}\right)$, the global equations are obtained with parts relative to static and dynamic balance equations. Stationary forces are equally separated from the dynamic forces:

$$
N=N_{s}+N_{t}, \quad V=V_{s}+V_{t}, \quad M=M_{s}+M_{t}
$$

this implies:

$$
N-N_{s}=N_{t}, \quad V-V_{s}=V_{t}, \quad M-M_{s}=M_{t}
$$

with $\left(N_{s}, M_{s}, V_{s}\right)$ are the static forces, $\left(N_{t}, M_{t}, V_{t}\right)$ are associated to the dynamic perturbation. Indeed, $\left(N_{t}, M_{t}, V_{t}\right)$ depend linearly from the dynamic displacement $\mathbf{u}_{t}$. They are obtained by subtracting the static forces of global efforts (70). Their detailed expressions are given in Appendix E.

\section{Inclusion of the rotation}

The general equations of equilibrium are written relatively to the rotating configuration from (32):

$$
\begin{aligned}
\rho \ddot{\mathbf{u}} & +2 \rho \operatorname{grad}_{r}(\dot{\mathbf{u}}) \cdot \boldsymbol{\Omega}\left(\mathbf{X}_{r}, t\right)+\rho \operatorname{grad}_{r}\left(\mathbf{F}_{r t} \cdot \boldsymbol{\Omega}\left(\mathbf{X}_{r}, t\right)\right) \cdot \boldsymbol{\Omega}\left(\mathbf{X}_{r}, t\right) \\
& =\rho \mathbf{f}+\operatorname{div}_{r}\left(\mathbf{F}_{r t} \cdot \mathbf{S}_{r}\right)
\end{aligned}
$$

with $\mathbf{u}$ is the global displacement vector such that $\mathbf{u}=\mathbf{u}_{s}+\mathbf{u}_{t}$. As in the precedent case, one integrates this formula on a piece of beam of length $d s$ 
along the neutral axis in the rotating configuration:

$$
\begin{aligned}
& \int_{\mathfrak{D}} \rho \ddot{\mathbf{u}} d V+\int_{\mathfrak{D}} 2 \rho \operatorname{grad}_{r}(\dot{\mathbf{u}}) \cdot \boldsymbol{\Omega}\left(\mathbf{X}_{r}, t\right) d V \\
& \quad+\int_{\mathfrak{D}} \rho \operatorname{grad}_{r}\left(\mathbf{F}_{r t} \cdot \boldsymbol{\Omega}\left(\mathbf{x}_{r}, t\right)\right) \cdot \boldsymbol{\Omega}\left(\mathbf{X}_{r}, t\right) d V=\int_{\mathcal{D}} \rho \mathbf{f}+\operatorname{div}_{r}\left(\mathbf{F}_{r t} \cdot \mathbf{S}_{r}\right) d V \\
& \int_{\mathfrak{D}} z \mathbf{t} \wedge \rho \ddot{\mathbf{u}} d V+\int_{\mathfrak{D}} z \mathbf{t} \wedge 2 \rho \operatorname{grad}_{r}(\dot{\mathbf{u}}) \cdot \boldsymbol{\Omega}\left(\mathbf{x}_{r}, t\right) d V \\
& \quad+\int_{\mathfrak{D}} z \mathbf{t} \wedge \rho \operatorname{grad}_{r}\left(\mathbf{F}_{r t} \cdot \boldsymbol{\Omega}\left(\mathbf{x}_{r}, t\right)\right) \cdot \boldsymbol{\Omega}\left(\mathbf{x}_{r}, t\right) d V=\int_{\mathfrak{D}} z \mathbf{t} \wedge\left(\rho \mathbf{f}+\operatorname{div}_{r}\left(\mathbf{F}_{r t} \cdot \mathbf{S}_{r}\right)\right) d V
\end{aligned}
$$

In equations (72), only the term for the gyroscopic effect has not yet been detailed. Using the computation of the gradient of the transformation (68), one has

$$
\operatorname{grad}_{r}(\dot{\mathbf{u}})=\left(\begin{array}{cc}
-\dot{\alpha}_{t} \sin \alpha & \frac{1}{R+z}\left(\dot{u}_{t}^{\prime}-\dot{w}_{t}-z \dot{\alpha}_{t}\left(1+\alpha^{\prime}\right) \cos \alpha-z \dot{\alpha}_{t}^{\prime} \sin \alpha\right) \\
\dot{\alpha}_{t} \cos \alpha & \frac{1}{R+z}\left(\dot{u}_{t}+\dot{w}_{t}^{\prime}-z \dot{\alpha}_{t}\left(1+\alpha^{\prime}\right) \sin \alpha+z \dot{\alpha}_{t}^{\prime} \cos \alpha\right)
\end{array}\right)
$$

With $\boldsymbol{\Omega}$ given by (58), this gives the gyroscopic term:

$$
\operatorname{grad}_{r}(\dot{\mathbf{u}}) \cdot \Omega\left(\mathbf{X}_{r}, t\right)=\Omega\left(\begin{array}{c}
\dot{u}_{t}^{\prime}-\dot{w}_{t}-z \dot{\alpha}_{t}\left(1+\alpha^{\prime}\right) \cos \alpha-z \dot{\alpha}_{t}^{\prime} \sin \alpha \\
\dot{u}_{t}+\dot{w}_{t}^{\prime}-z \dot{\alpha}_{t}\left(1+\alpha^{\prime}\right) \sin \alpha+z \dot{\alpha}_{t}^{\prime} \cos \alpha
\end{array}\right)
$$

Finally:

$$
\left\{\begin{array}{l}
\int_{\mathcal{D}} 2 \rho \operatorname{grad}_{r}(\dot{\mathbf{u}}) \cdot \boldsymbol{\Omega}\left(\mathbf{x}_{r}, t\right) d V=2 \rho \Omega A\left(\dot{u}_{t}^{\prime}-\dot{w}_{t}\right) \mathbf{u}_{R}+2 \rho \Omega A\left(\dot{u}_{t}+\dot{w}_{t}^{\prime}\right) \mathbf{u}_{\theta} \\
\int_{\mathcal{D}} z \cdot \mathbf{t} \wedge 2 \rho \operatorname{grad}_{r}(\dot{\mathbf{u}}) \cdot \boldsymbol{\Omega}\left(\mathbf{x}_{r}, t\right) d V=2 \rho \Omega I \dot{\alpha}_{t}^{\prime}
\end{array}\right.
$$

The equations of equilibrium of the case without rotation (E.2) are com- 
pleted by adding terms due to the rotation:

$$
\begin{aligned}
& \frac{1}{R}\left(V_{t}^{\prime} \cos \alpha-N_{t}^{\prime} \sin \alpha-\alpha_{t}^{\prime}(V \sin \alpha+N \cos \alpha)\right)+p \frac{u_{t}+w_{t}^{\prime}}{R}+p_{t} \frac{R+u+w^{\prime}}{R} \\
& -\frac{\alpha_{t}}{R}\left(V^{\prime} \sin \alpha+N^{\prime} \cos \alpha\right) \\
& -\frac{\alpha^{\prime}+1}{R}\left(V_{t} \sin \alpha+N_{t} \cos \alpha+\alpha_{t}(V \cos \alpha-N \sin \alpha)\right) \\
& +f_{R}{ }^{t}-k_{R} u_{t}-\rho \Omega^{2} A\left(u_{t}^{\prime \prime}-2 w_{t}^{\prime}-u_{t}\right)=\rho A \ddot{u}_{t}+2 \rho \Omega A\left(\dot{u}_{t}^{\prime}-\dot{w}_{t}\right) \\
& \frac{1}{R}\left(V_{t}^{\prime} \sin \alpha+N_{t}^{\prime} \cos \alpha+\alpha_{t}^{\prime}(V \cos \alpha-N \sin \alpha)\right)-p \frac{u_{t}^{\prime}-w_{t}}{R}-p_{t} \frac{u^{\prime}-w}{R} \\
& +\frac{\alpha_{t}}{R}\left(V^{\prime} \cos \alpha-N^{\prime} \sin \alpha\right) \\
& +\frac{\alpha^{\prime}+1}{R}\left(V_{t} \cos \alpha-N_{t} \sin \alpha-\alpha_{t}(N \cos \alpha+V \sin \alpha)\right) \\
& -k_{\theta} w-\rho \Omega^{2} A\left(w_{t}^{\prime \prime}+2 u_{t}^{\prime}-w_{t}\right)=\rho A \ddot{w}_{t}+2 \rho \Omega A\left(\dot{u}_{t}+\dot{w}_{t}^{\prime}\right) \\
& \frac{1}{R} M_{t}^{\prime}+\frac{1}{R}\left(\left(u_{t}^{\prime}-w_{t}\right)(V \sin \alpha+N \cos \alpha)-\left(u_{t}+w_{t}^{\prime}\right)(V \cos \alpha-N \sin \alpha)\right)+ \\
& \frac{1}{R}\left(\left(u^{\prime}-w\right)\left(V_{t} \sin \alpha+N_{t} \cos \alpha\right)-\left(R+u+w^{\prime}\right)\left(V_{t} \cos \alpha-N_{t} \sin \alpha\right)\right)+ \\
& \frac{1}{R}\left(\alpha_{t}\left(u^{\prime}-w\right)(V \cos \alpha-N \sin \alpha)+\alpha_{t}\left(R+u+w^{\prime}\right)(V \sin \alpha+N \cos \alpha)\right) \\
& -\rho \Omega^{2} I \alpha_{t}^{\prime \prime}=\rho I \ddot{\alpha}_{t}+2 \rho \Omega I \dot{\alpha}_{t}^{\prime}
\end{aligned}
$$

\subsubsection{Construction of the numerical equilibrium equations}

One also proposes a numerical method to solve the ordinary differential equations (61). The stationary equilibrium equations are written under the form:

$$
\mathbf{g}\left(\mathbf{u}_{s}\right)=\mathbf{f}_{s}
$$

with $\mathbf{g}$ represents the function of the internal forces in the beam which are not always linear with the displacement $\mathbf{u}_{s}$. By adding a small disturbance $\mathbf{u}_{t}$, the dynamic relation becomes:

$$
\mathbf{M} \ddot{\mathbf{u}}_{t}+\mathbf{C} \dot{\mathbf{u}}_{t}+\mathbf{g}\left(\mathbf{u}_{s}+\mathbf{u}_{t}\right)=\mathbf{f}_{s}+\mathbf{f}_{t}
$$

Equation (78) is linearized around $\mathbf{u}_{t}$. One has:

$$
\mathbf{g}\left(\mathbf{u}_{s}+\mathbf{u}_{t}\right)=\mathbf{g}\left(\mathbf{u}_{s}\right)+\frac{\partial \mathbf{g}}{\partial \mathbf{u}_{s}} \mathbf{u}_{t}
$$

Replacing (77) and (79) in equation (78):

$$
\mathbf{M} \ddot{\mathbf{u}}_{t}+\mathbf{C} \dot{\mathbf{u}}_{t}+\frac{\partial \mathbf{g}}{\partial \mathbf{u}_{s}} \mathbf{u}_{t}=\mathbf{f}_{t}
$$

Setting:

$$
\frac{\partial \mathbf{g}}{\partial \mathbf{u}_{s}}=\mathbf{K}
$$


Finally, the numerical equations are as follows:

$$
\mathbf{M} \ddot{\mathbf{u}}_{t}+\mathbf{C} \dot{\mathbf{u}}_{t}+\mathbf{K} \mathbf{u}_{t}=\mathbf{f}_{t}
$$

The matrix $\mathbf{K}$ is found by the stationary solution. We still have to find two matrices $\mathbf{M}$ and $\mathbf{C}$. In fact, the matrix $\mathbf{M}$ is easily constructed by discretizing the term of the relative acceleration in the dynamic equation (76). The matrix $\mathbf{C}$ is the gyroscopic matrix which is also constructed by discretizing the analytical equations (76).

\section{Validation of models and rotating effects}

\subsection{Stationary case}

The system of differential equations of the second order (61) has periodical boundary conditions:

$$
\left\{\begin{array}{l}
\mathbf{u}=\mathbf{f}\left(\theta, \mathbf{u}, \mathbf{u}^{\prime}\right) \\
\mathbf{u}(0)=\mathbf{u}(2 \pi) \\
\mathbf{u}^{\prime}(0)=\mathbf{u}^{\prime}(2 \pi)
\end{array}\right.
$$

with $\mathbf{u}$ is the displacement vector. The exact solution is approximated by a discrete solution. For this, a mesh of $N$ points is defined with $\theta=\left(\theta_{1}=\right.$ $\left.0 ; \theta_{2} ; \ldots \theta_{i} \ldots ; \theta_{N}=2 \pi(N-1) / N\right)$. The discretized values are:

$$
\left\{\begin{array}{l}
\mathbf{u}^{\prime}\left(\theta_{i}\right)=\frac{\mathbf{u}\left(\theta_{i+1}\right)-\mathbf{u}\left(\theta_{i-1}\right)}{2 h}=\frac{\mathbf{u}_{i+1}-\mathbf{u}_{i-1}}{2 h} \\
\mathbf{u}^{\prime \prime}\left(\theta_{i}\right)=\frac{\mathbf{u}\left(\theta_{i+1}\right)-2 \mathbf{u}\left(\theta_{i}\right)+\mathbf{u}\left(\theta_{i-1}\right)}{h^{2}}=\frac{\mathbf{u}_{i+1}-2 \mathbf{u}_{i}+\mathbf{u}_{i-1}}{h^{2}} \\
\mathbf{u}^{\prime \prime}\left(\theta_{i}\right)=f\left(\theta_{i}, \mathbf{u}\left(\theta_{i}\right), \mathbf{u}^{\prime}\left(\theta_{i}\right)\right) \leftrightarrow \frac{\mathbf{u}_{i+1}-2 \mathbf{u}_{i}+\mathbf{u}_{i-1}}{h^{2}}=f\left(\theta_{i}, \mathbf{u}_{i}, \frac{\mathbf{u}_{i+1}-\mathbf{u}_{i-1}}{2 h}\right)
\end{array}\right.
$$

The boundary conditions are written as:

$$
\mathbf{u}(0)=\mathbf{u}(2 \pi) \rightarrow\left\{\begin{array}{l}
\mathbf{u}_{1}{ }^{\prime}=\frac{\mathbf{u}_{2}-\mathbf{u}_{N}}{2 h} ; \mathbf{u}_{N}{ }^{\prime}=\frac{\mathbf{u}_{1}-\mathbf{u}_{N-1}}{2 h} \\
\mathbf{u}_{1}{ }^{\prime \prime}=\frac{\mathbf{u}_{2}-2 \mathbf{u}_{1}+\mathbf{u}_{N}}{h^{2}} ; \mathbf{u}_{N}{ }^{\prime \prime}=\frac{\mathbf{u}_{1}-2 \mathbf{u}_{N}+\mathbf{u}_{N-1}}{h^{2}}
\end{array}\right.
$$

The relations (83) and (84) provide $N$ equations with $N$ unknowns. In the linear case, finding $\mathbf{u}$ is immediate. For the nonlinear case, the Newton method is used. The model parameters are shown in the table (2).

\subsection{Dynamic case}

The dynamic equations are written in the form:

$$
\mathbf{M} \ddot{\mathbf{u}}_{t}+\mathbf{C} \dot{\mathbf{u}}_{t}+\mathbf{K} \mathbf{u}_{t}=\mathbf{f}_{t}
$$

The matrices $\mathbf{M}$ and $\mathbf{C}$ are constructed directly using analytical equations. In the case of the linear static deformation, stiffness matrix $\mathbf{K}$ does not change during the deformation. It is built directly by the analytical equations without rotation or with rotation. In the case where the effect of large 


\begin{tabular}{|c|c|c|c|}
\hline Parameter & Description & Value & Unit \\
\hline$E$ & Young modulus & $2.6611 e 9$ & $\mathrm{~Pa}$ \\
$\rho$ & Density & 1160.7 & $\mathrm{kgm}^{-3}$ \\
$R$ & Ring radius & 0.285 & $\mathrm{~m}$ \\
$e$ & Thickness of the beam & 0.01 & $\mathrm{~m}$ \\
$b$ & Width of the beam & 0.085 & $\mathrm{~m}$ \\
$k_{R}$ & Radial stiffness & $4.35 e 6$ & $\mathrm{~Pa}$ \\
$k_{\theta}$ & Tangential stiffness & $3.19 e 5$ & $\mathrm{~Pa}$ \\
$\nu$ & Poisson coefficient & 0.3 & \\
\hline
\end{tabular}

Table 2: Model parameters

displacements is taken into account, the stiffness matrix varies during the deformation. Stiffness matrix obtained at the end of the static state is the one considered in the dynamic calculation. There are two ways to calculate this matrix either using analytical equations using the stationary solutions, or as the Jacobian of stationary equations (see section 3) calculated by numerical solution of the stationary case. In order to validate the analytical model of a circular ring, it is compared to the result of the simulation of this model with Abaqus.

\subsection{D FEM with beams}

This model is built to validate the analytical formulations of the circular ring model. It does not take into account the effect of rotation. The parameters and loading conditions are identical to the analytical model. In the finite element code Abaqus, one uses the Euler Bernoulli beam elements (B23 element), and Timoshenko beams (B21 element). The ring is discretized in $N$ nodes each connected to two springs: a radial spring and a tangential spring. The values of the stiffness of the springs are calculated from the analytical stiffness springs as follows:

$$
\left\{\begin{array}{l}
k_{R}^{\text {num }}=R \triangle \theta k_{R} \\
k_{\theta}^{\text {num }}=R \triangle \theta k_{\theta}
\end{array}\right.
$$

\subsection{D FEM with shells}

To take into account the effects of rotation in the circular ring model, the *STEADY STATE TRANSPORT functionality available under Abaqus is used. It can be applied, however, only on shells or solid elements. It is necessary for this reason to build a similar model based on shell elements. The parameters of the model geometry do not change (Figure 5). The radial and tangential springs are also retained. A static validation is performed before any application of rotational effects. 


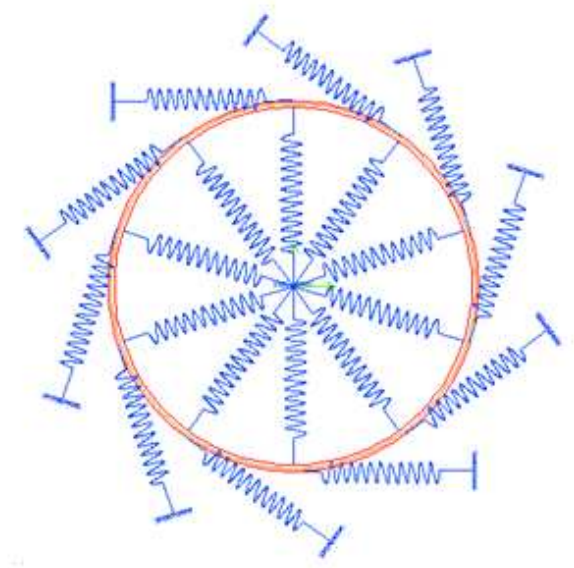

Figure 5: Circular ring model with Abaqus

\subsection{Case without rotation, analytical model versus FEM with beams}

The results of the steady state between the Matlab calculation and the calculation under Abaqus are compared at constant boundary conditions and equivalent load.

\section{Vehicle load}

Vehicle load is applied to the wheel centre. It is represented by a point force imposed at the base of the tire. The comparison between the Matlab modelling and Abaqus simulation is shown in Figure 6. A good correlation of the displacements is observed. Note that the results of the Euler Bernoulli beam and those of the Timoshenko beam are identical. This is explained by the use of the assumption of the thin beam (the shearing effect is neglected).

2. Comparison of deformed linear and nonlinear configurations A comparison of the deformed configurations of the linear case and the nonlinear case in geometry is shown in Figure 7. There are differences around the excitation point. Near this point one can expect that the stress and strains are large and so in this zone the differences between the linear and non linear models will be the largest.

\section{Modes of vibration}

The natural frequencies of the tire in its deformed configuration are compared. Two types of boundary conditions are considered: fixed wheel centre / free tire base and both fixed wheel centre and tire base. The results obtained with Matlab and Abaqus are very close. The differences are less than $1.2 \%$ which is satisfactory. Mode shapes are also identical. In the case where the tire base is free, ring symmetry is preserved. In this case, double modes at the same frequency are 


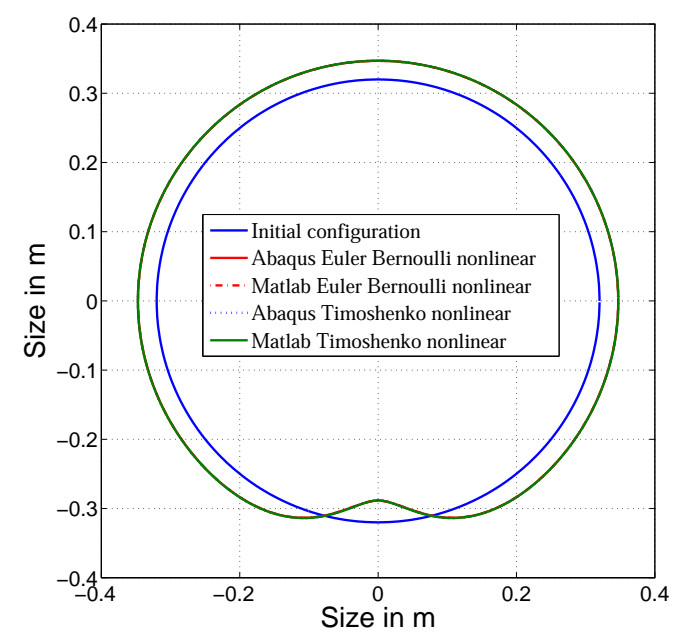

Figure 6: Undeformed and deformed configurations of the tire resulting from nonlinear static calculations for a point force at the bottom of the tire

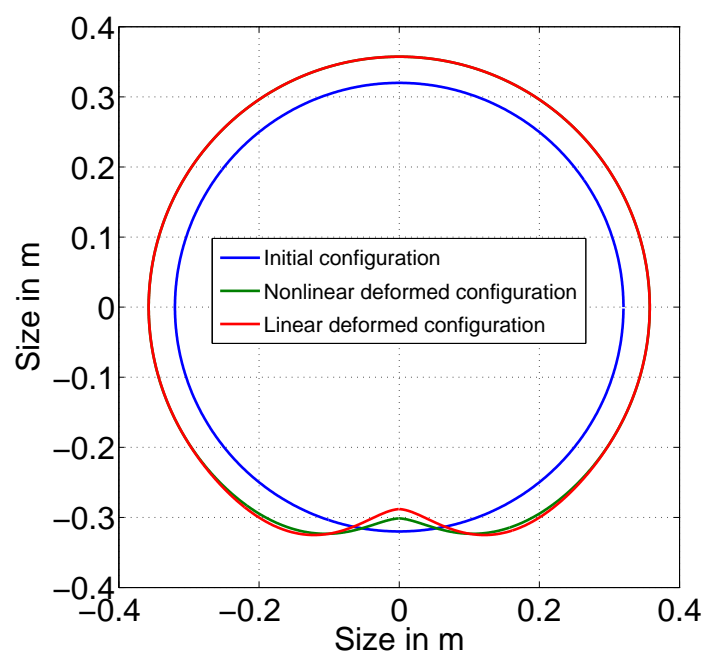

Figure 7: Undeformed and deformed configurations of the tire due to linear and nonlinear static calculations for a point force at the bottom of the tire

observed and obtained one from the other by a rotation of $\frac{\pi}{2 n}$ (Figure 8). In contrast, if the tire base is fixed, this property is lost (Figure 9 ). These observations are identical to what was found for instance by [33] in the case of a FEM modelling. The frequency range of these modes is quite similar to what is reported in [29]. In conclusion, the 
analytical model is validated up to $400 \mathrm{~Hz}$.

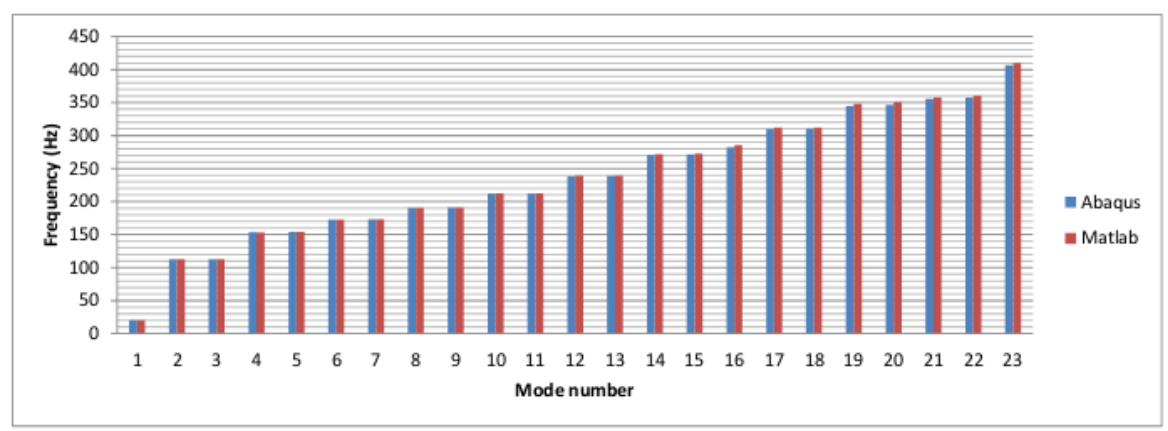

Figure 8: Eigenmodes with a fixed wheel centre and a free tire base

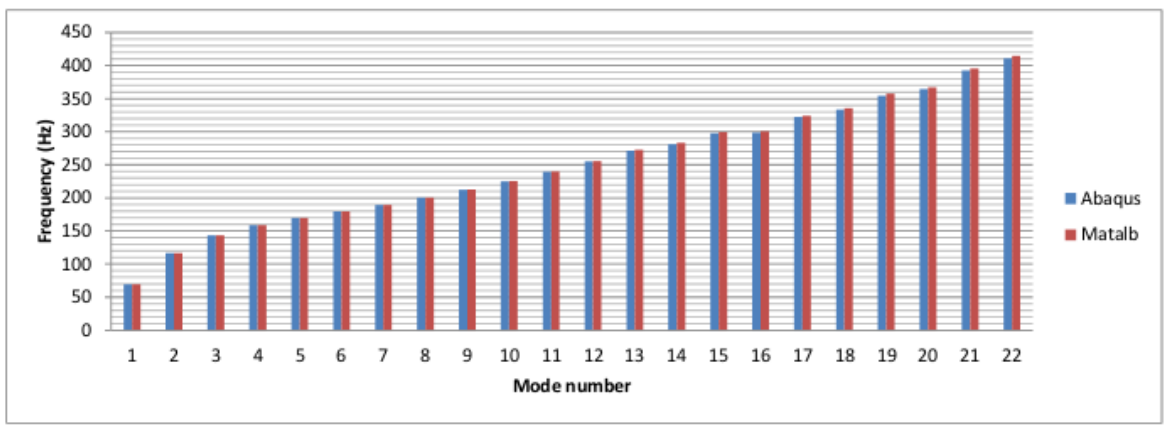

Figure 9: Eigenmodes with fixed wheel centre and tire base

\section{Transfer functions}

An example of transfer function is shown in Figure 10. It is the vertical reaction force at the centre resulting from a vertical dynamic force applied in the tire base with constant unit amplitude. The vertical reaction force at the wheel centre which is the sum of the vertical reactions of the radial and tangential springs, is obtained as follows:

$$
R_{y}=\int_{0}^{2 \pi} k_{R} u_{t} \cdot\left(\mathbf{u}_{R}, \mathbf{y}\right) d \theta+\int_{0}^{2 \pi} k_{\theta} w_{t} \cdot\left(\mathbf{u}_{\theta}, \mathbf{y}\right) d \theta
$$

with $($,$) means the scalar product in \Re^{2}$. Matlab and Abaqus curves are superposed up to $300 \mathrm{~Hz}$ and have small differences in the frequencies around $350 \mathrm{~Hz}$ because the natural frequencies calculated in Matlab and Abaqus are slightly different, $f_{20}=346 \mathrm{~Hz}$ under Abaqus and $f_{20}=350 \mathrm{~Hz}$ under Matlab (cf. figure 10). The difference is very low and we get a good agreement. The agreement between two different computations with different software makes us confident into the 
validity of these results. Moreover the experimental results reported in [30] show similar tendencies with a maximum near $100 \mathrm{~Hz}$ and values extending over a range of about $30 \mathrm{~dB}$.

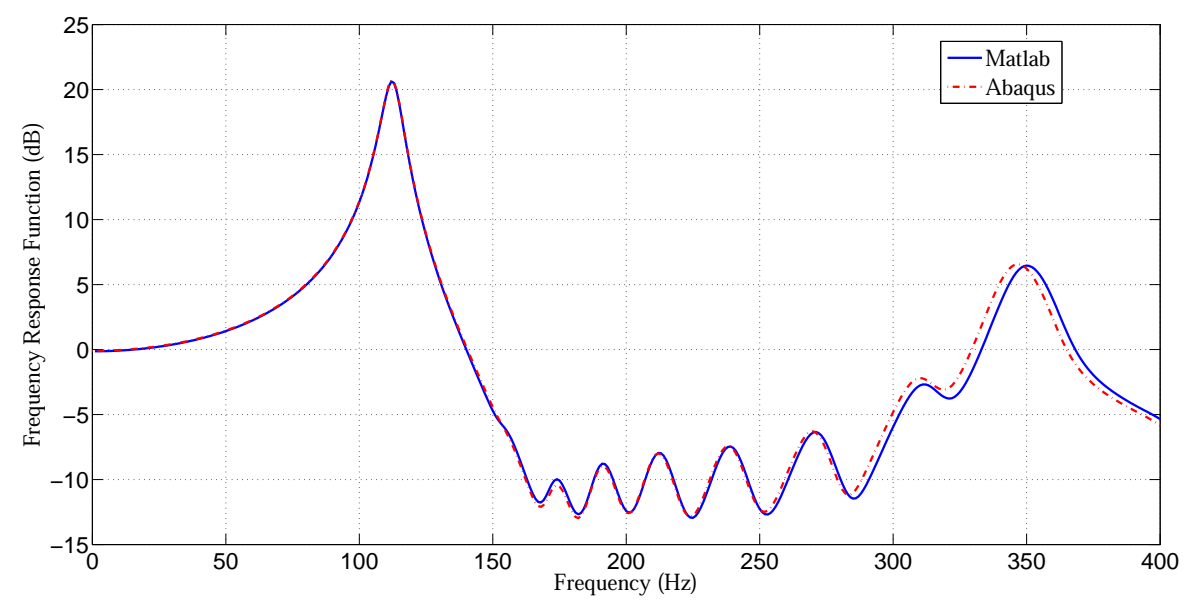

Figure 10: Response of the vertical force at the wheel centre for an excitation at the base of the tire for the case with no-rotation and no-load except the dynamic excitation

\subsection{Case with rotation, analytical model versus shell FEM}

In section 3, the development of analytical formulations showed that taking into account the rotating effects consists in adding a centripetal stiffness matrix and a viscous damping matrix. The effect on the change in the stiffness of the structure is very small. On the contrary, the gyroscopic damping strongly changes the dynamic response and causes a frequency splitting according to the speed of rotation. This is shown by the theoretical work of Bolton and Kim [18] and the experimental works of [30,6,31]. This result is confirmed in this section.

\section{Linear deformation case}

Regarding the linear case, the natural frequencies are calculated analytically. Meanwhile, a calculation can be performed using the numerical equations in the frequency domain:

$$
-\tilde{\omega}_{n}^{2} \mathbf{M} \mathbf{u}_{n}+i \tilde{\omega}_{n} \mathbf{C} \mathbf{u}_{n}+\mathbf{K} \mathbf{u}_{n}=\mathbf{0}
$$

with $\mathbf{u}_{n}$ is the displacement vector of the ring.

Denoting $\tilde{\mathbf{u}}_{n}=\left(\begin{array}{c}\mathbf{u}_{n} \\ i \tilde{\omega}_{n} \mathbf{u}_{n}\end{array}\right)$ the complex eigenvector equations become:

$$
i \tilde{\omega}_{n}\left(\begin{array}{cc}
\mathbf{K} & \mathbf{0} \\
\mathbf{0} & \mathbf{M}
\end{array}\right)\left(\begin{array}{c}
\mathbf{u}_{n} \\
i \tilde{\omega}_{n} \mathbf{u}_{n}
\end{array}\right)+\left(\begin{array}{cc}
\mathbf{0} & -\mathbf{K} \\
\mathbf{K} & \mathbf{C}
\end{array}\right)\left(\begin{array}{c}
\mathbf{u}_{n} \\
i \tilde{\omega}_{n} \mathbf{u}_{n}
\end{array}\right)=\left(\begin{array}{l}
\mathbf{0} \\
\mathbf{0}
\end{array}\right)
$$


So, the numerical frequency $\tilde{\omega}_{n}$ is given by:

$$
\operatorname{det}\left\|i \tilde{\omega}_{n}\left(\begin{array}{cc}
\mathbf{K} & \mathbf{0} \\
\mathbf{0} & \mathbf{M}
\end{array}\right)+\left(\begin{array}{cc}
\mathbf{0} & -\mathbf{K} \\
\mathbf{K} & \mathbf{C}
\end{array}\right)\right\|=0
$$

When the matrices $\mathbf{M}$ and $\mathbf{K}$ are symmetric and the matrix $\mathbf{C}$ is skew-symmetric, the first matrix on the left of the equation (90) is symmetrical and the second is skew-symmetric. It is easy to show that the eigenvalues are imaginary. This implies that the eigenfrequencies of the system are real. One checks that the natural frequencies calculated by the analytical and numerical models at different speeds are very close. It is shown that the natural frequencies depend on the speed of rotation. This effect is illustrated in Figure 11.

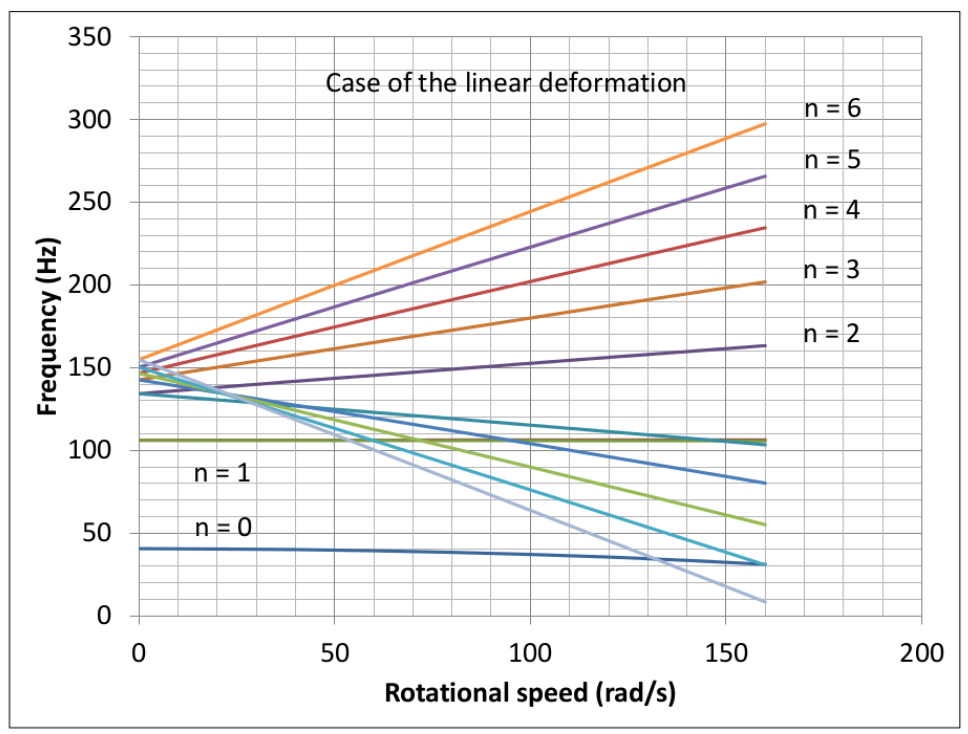

Figure 11: Frequencies versus the rotation speed for the undeformed tire

\section{Nonlinear deformation case}

Having no simple analytical solution, a direct comparison of numerical results with Matlab and Abaqus is realized. As above, the dynamic solutions of the tire are compared around the deformed stationary configuration under a load with the parameters: circular velocity $\Omega=$ $100 \mathrm{rad} / \mathrm{s}$, inflation pressure $p=2.5 \mathrm{bar}$ and a vehicle load $q=250 \mathrm{~kg}$. A good correlation of natural frequencies calculated in Matlab and Abaqus is observed, as shown in Figure 12. The gap increases with frequency. However, in the range $[0,400 \mathrm{~Hz}]$, the maximum deviation is $7.3 \%$ which remains moderate. In conclusion, the analytical model in nonlinear deformation is validated for low frequencies. 
As for the case without rotation (Figure 10), dynamic responses at the wheel centre are compared. The formulation (87) is repeated for assessing the vertical reaction. From the deformed configuration, a harmonic force $1 e^{i \omega t}$ is imposed at the tire base. The response is plotted versus the frequency. Under Abaqus, the mass matrix and the stiffness matrix are extracted from the model. Using these matrices, calculation of the transfer function is performed using Matlab.

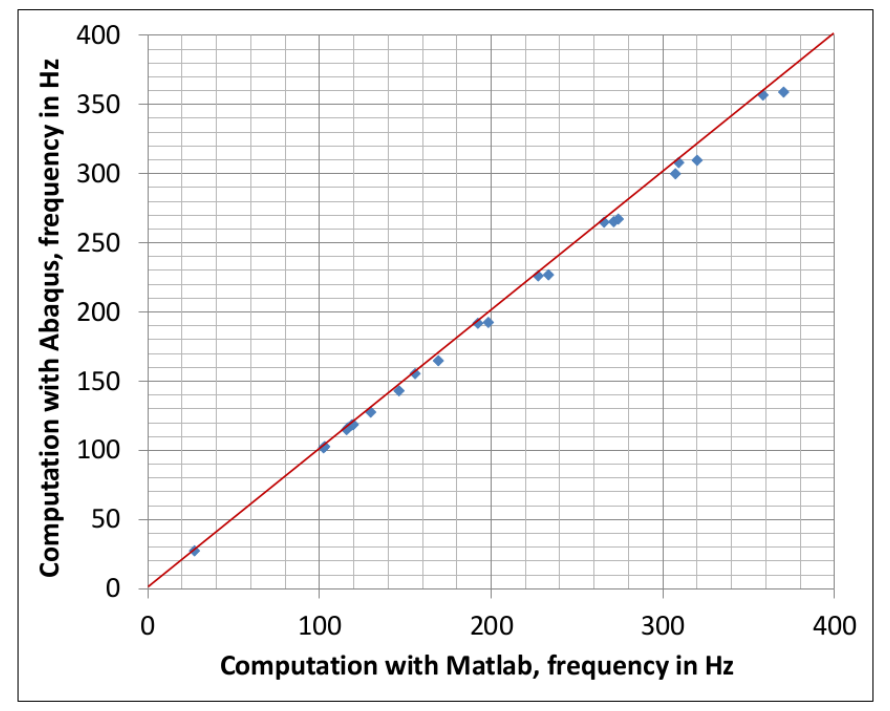

Figure 12: Eigenfrequencies of the circular ring model at $\Omega=100 \mathrm{rad} / \mathrm{s}$ in the nonlinear deformation case for a load of $250 \mathrm{~kg}$ applied at the bottom of tire

Figure 13 shows the comparison of the transfer functions. At low frequencies, the results are very close. Gaps begin to appear from $250 \mathrm{~Hz}$. Concerning the rotation effect, the transfer functions versus the speed of rotation are plotted in Figures 14(a) and 14(b). The first resonance corresponds to the frequency $112 \mathrm{~Hz}$ of the "spring" mode and there is no duplication. The explanation is found by the absence of mass of springs. One can see different behaviours near 350 Hz. This frequency corresponds to the eighth mode. For the vertical force, two maxima are observable for low frequencies. This is explained by looking at the deformed configuration of this mode. In fact, this mode is a combination of two tire vibrations: a radial vibration and a vibration in traction and compression tangent to the neutral fiber. The radial vibration has eight peaks on the deformed configuration. The other has two vibration maximums. This is why we see two peaks on the vertical response curve. The tension-compression vibration like 


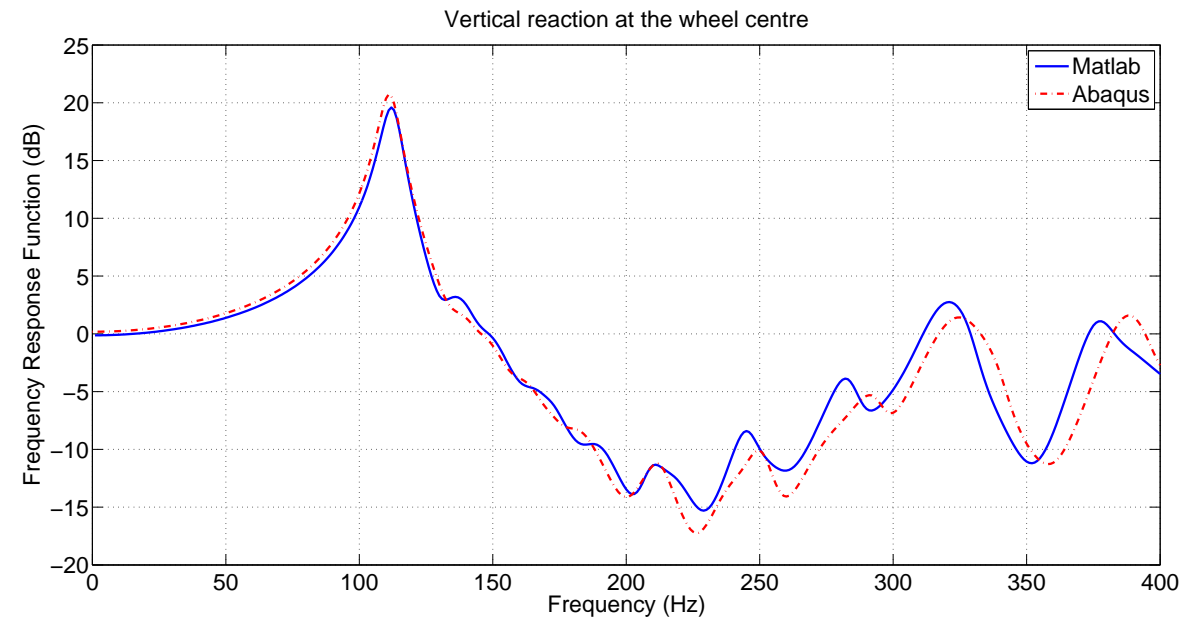

Figure 13: Ring response for the forced excitation at $(\Omega=100 \mathrm{rad} / \mathrm{s})$ for a load of $250 \mathrm{~kg}$ applied at the bottom of the tire

mode is symmetric, so it does not excite the horizontal force. This is reason why we do not observe the second peak on the horizontal response curve.

To observe the frequency splitting effect, we construct a map of the transfer function at the wheel centre versus the speed. Figure 15 represents the vertical reaction at the wheel centre for a tire loaded with a vertical force of $250 \mathrm{~kg}$ applied at the bottom of the tire. The horizontal axis is the frequency and the vertical axis is the linear velocity. The maps built with Matlab and Abaqus are very similar which validates the circular ring model with rotating effect. The splitting according to the speed is seen in the form of two branches from zero speed. The spacing between the arms is a function of the wave number. It only affects the modes including the deformation of the tread. For the first mode, the tread does not deform, so there is no duplication. If we compare this figure with figure 11, we can see that similar behaviours are observed in the deformed and undeformed cases. The mode at the frequency $100 \mathrm{~Hz}$ do no change with the rotating velocity. On the contrary there is a splitting of modes associated with higher frequencies.

\section{Conclusion}

In this paper, a 2D nonlinear ring model for the analysis of tire dynamic behaviour in both non-rolling and rolling conditions is proposed. The assumptions of Timoshenko beam and finite displacements are considered to build the model. Thus, large rotations of the cross section and high order of translation displacements are taken into account but the cross section is 


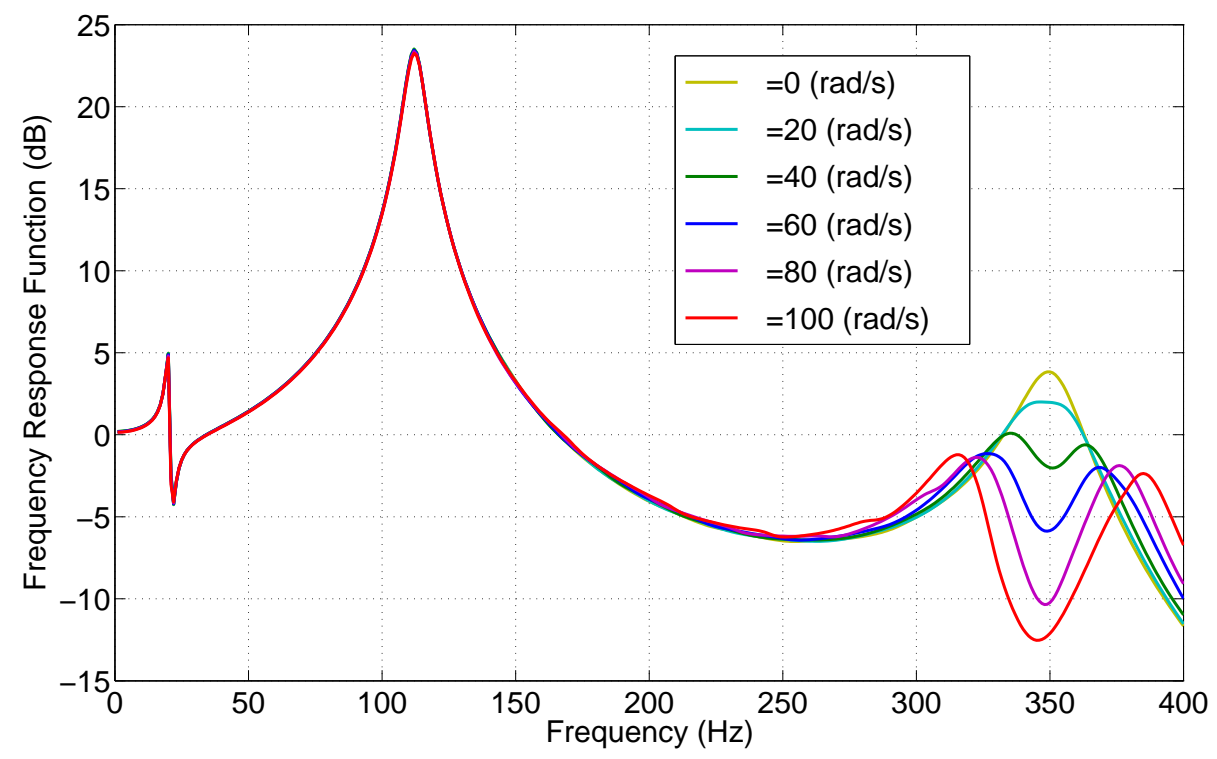

(a) Horizontal reaction

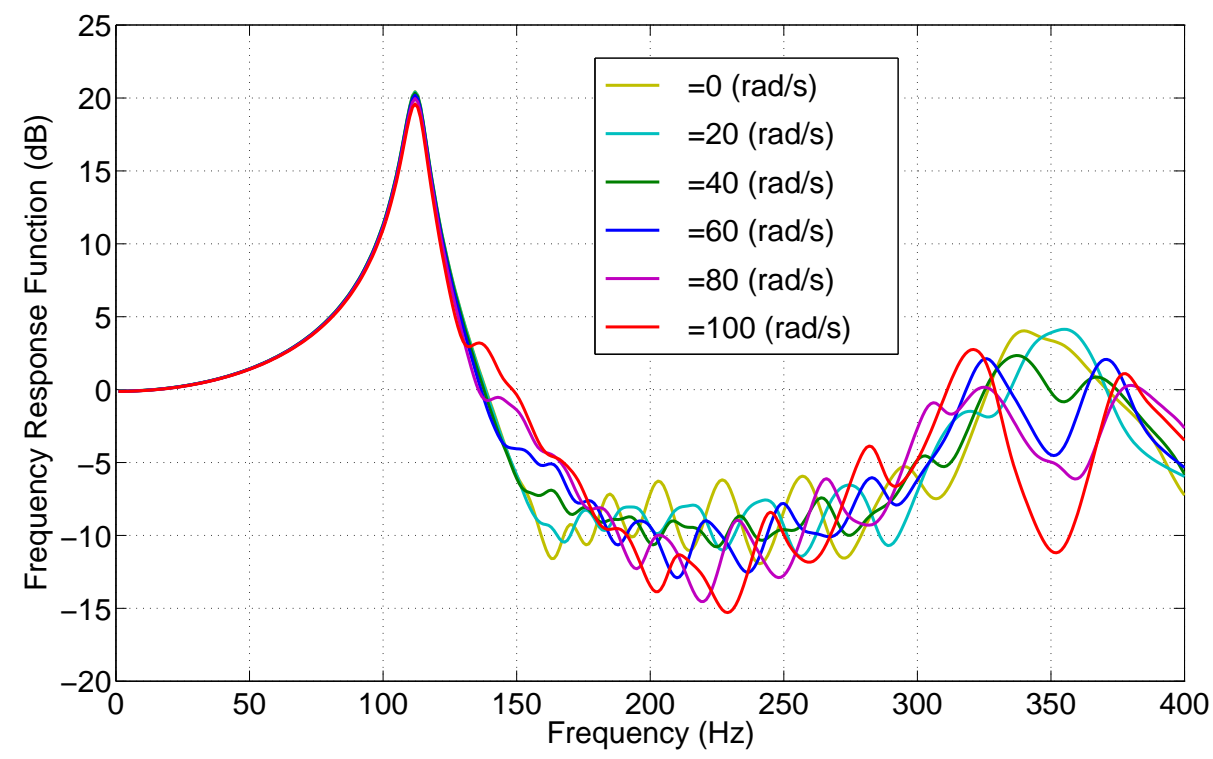

(b) Vertical reaction

Figure 14: The longitudinal and vertical responses at the wheel centre for a vehicle load of $250 \mathrm{~kg}$

assumed to remain non-deformed. The analytical formulation is established successfully in linear/nonlinear static and dynamic states in non-rolling and 


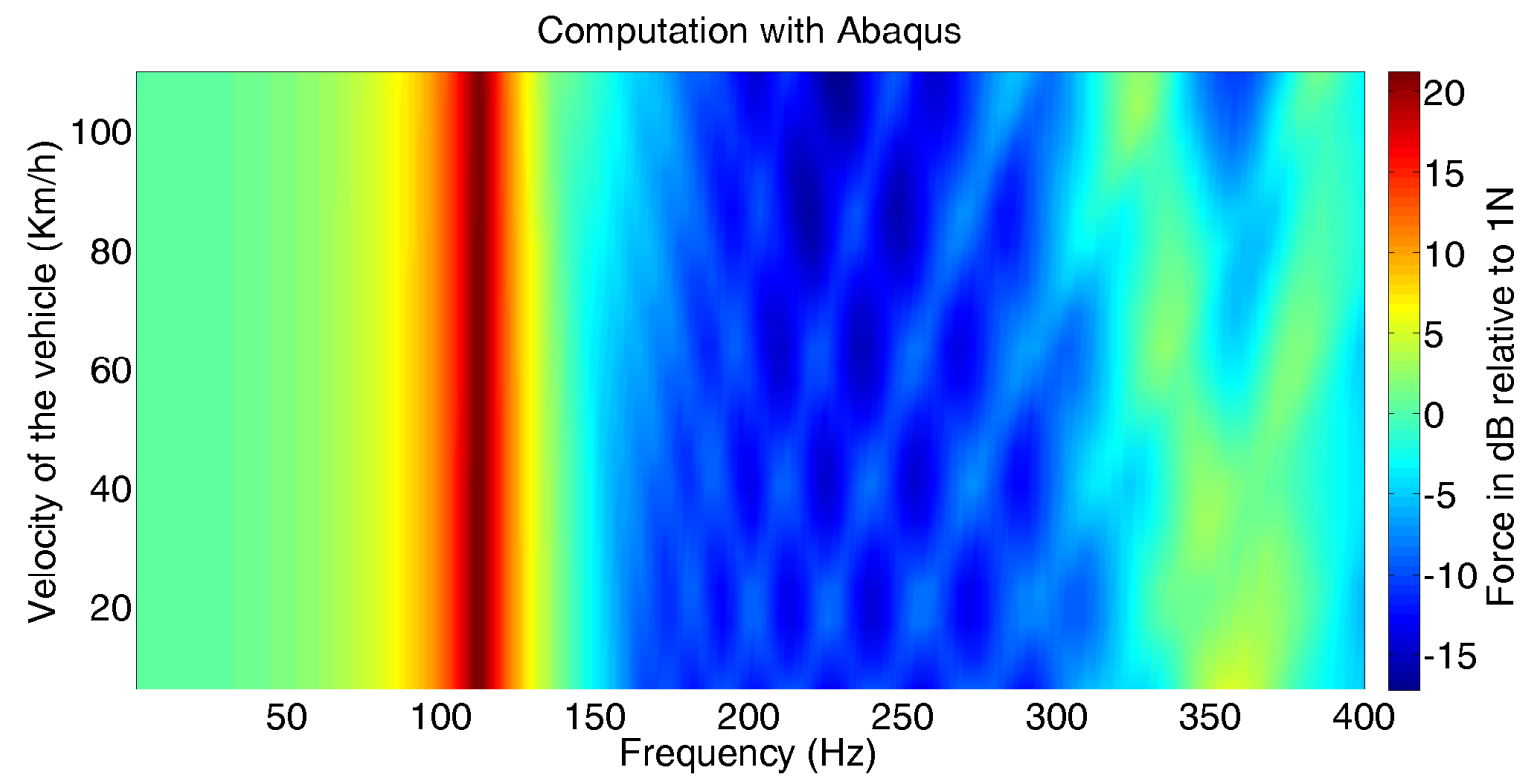

(a)

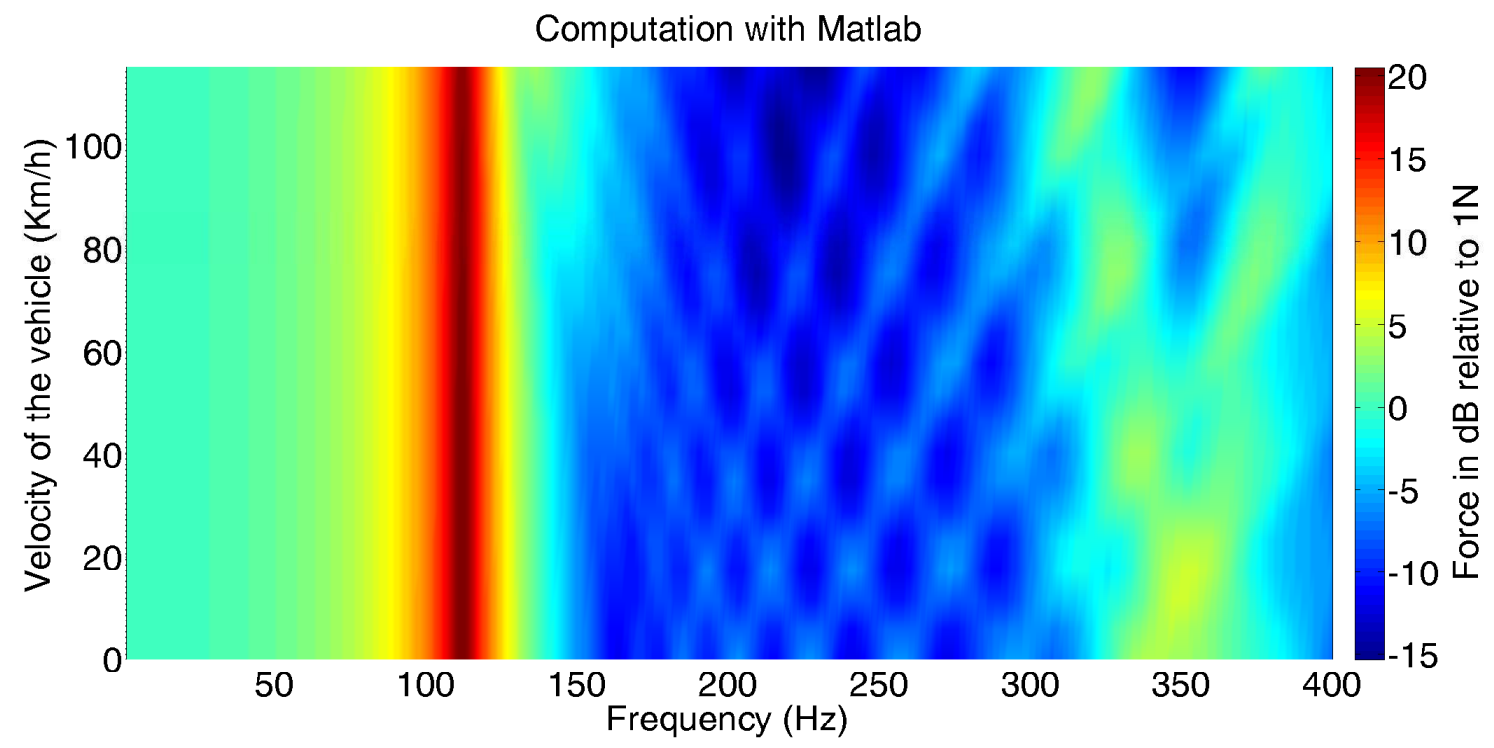

(b)

Figure 15: Mapping vertical reaction at the wheel center in Abaqus (a) and Matlab (b) for the deformed tire with a $250 \mathrm{~kg}$ load at the bottom

rolling conditions using an Arbitrary Lagrangian Eulerian approach. Since the nonlinear problem cannot be solved analytically, a numerical resolution 
method by discretization is proposed. The comparison with Abaqus simulations considered as reference allows the validation of the model and the rotating effects. First, in the static case, we check that the deformed shapes are similar. In the dynamic case without rotation, we verify that the eigenmodes and transfer functions computed around the static state are in good agreement. In rolling conditions, the dynamic behaviour can also be pre-

dicted in a satisfactory manner. One finds the rotating effects as splitting of natural frequencies with velocity.

\section{Acknowledgements}

We thank the anonymous reviewers for valuable comments and suggestions which greatly improved the quality of this article. 


\section{Appendix A. Strain tensor}

To establish the relationship between $d \mathbf{u}$ and $d \mathbf{O P}$, equation (33) is taken to calculate:

$$
d \mathbf{O P}=d\left((R+z) \mathbf{u}_{R}\right)=d(R+z) \mathbf{u}_{R}+(R+z) d \mathbf{u}_{R}=d z \mathbf{u}_{R}+(R+z) d \theta \mathbf{u}_{\theta}
$$

This allows to write:

$$
d z=\mathbf{u}_{R} \cdot d \mathbf{O P} ; \quad d \theta=\frac{1}{R+z} \mathbf{u}_{\theta} \cdot d(\mathbf{O P})
$$

From relation (35), one also has:

$$
d \mathbf{u}=d\left((u+z(\cos \alpha-1)) \mathbf{u}_{R}+(w+z \sin \alpha) \mathbf{u}_{\theta}\right)
$$

Developing:

$$
\begin{aligned}
d \mathbf{u}=\left(d z(\cos \alpha-1)+\left(u^{\prime}-z \alpha^{\prime} \sin \alpha\right) d \theta-(w+z \sin \alpha) d \theta\right) \mathbf{u}_{R} \\
+\left(d z \sin \alpha+(u+z(\cos \alpha-1)) d \theta+\left(w^{\prime}+z \alpha^{\prime} \cos \alpha\right) d \theta\right) \mathbf{u}_{\theta}
\end{aligned}
$$

where exponent ()$^{\prime}$ means the derivation relative to $\theta$, i.e. ()$^{\prime}=\frac{\partial}{\partial \theta}$.

Using (A.1), the expression becomes:

$$
\begin{gathered}
d \mathbf{u}=\left((\cos \alpha-1) \mathbf{u}_{R} \otimes \mathbf{u}_{R}+\frac{1}{R+z}\left(u^{\prime}-z \alpha^{\prime} \sin \alpha-w-z \sin \alpha\right) \mathbf{u}_{R} \otimes \mathbf{u}_{\theta}+\right. \\
\left.\sin \alpha \mathbf{u}_{\theta} \otimes \mathbf{u}_{R}+\frac{1}{R+z}\left(u+z(\cos \alpha-1)+\left(w^{\prime}+z \alpha^{\prime} \cos \alpha\right)\right) \mathbf{u}_{\theta} \otimes \mathbf{u}_{\theta}\right) . d \mathbf{O P}
\end{gathered}
$$

with $\otimes$ is the tensor product. The gradient of the displacement field can be written in matrix form in the reference system $\left(\mathbf{u}_{R}, \mathbf{u}_{\theta}\right)$ :

$$
\operatorname{grad}(\mathbf{u})=\left(\begin{array}{cc}
\cos \alpha-1 & \frac{1}{R+z}\left(u^{\prime}-w-z \alpha^{\prime} \sin \alpha-z \sin \alpha\right) \\
\sin \alpha & \frac{1}{R+z}\left(u+w^{\prime}+z\left(\cos \alpha+\alpha^{\prime} \cos \alpha-1\right)\right)
\end{array}\right)
$$

The tensor of the gradient of the transformation is calculated by:

$$
\begin{aligned}
\mathbf{F} & =\mathbf{I}+\operatorname{grad}(\mathbf{u}) \\
& =\left(\begin{array}{cc}
\cos \alpha & \frac{1}{R+z}\left(u^{\prime}-w-z \alpha^{\prime} \sin \alpha-z \sin \alpha\right) \\
\sin \alpha & \frac{1}{R+z}\left(R+u+w^{\prime}+z\left(\cos \alpha+\alpha^{\prime} \cos \alpha\right)\right)
\end{array}\right)
\end{aligned}
$$


Denoting $\zeta=\frac{z}{R}$, the so-called thin ring condition is written as $\zeta \ll 1$. The tensor of the transformation is thus:

$$
\mathbf{F}=\left(\begin{array}{cc}
\cos \alpha & \frac{1}{1+\zeta}\left(\frac{u^{\prime}-w}{R}-\zeta\left(\alpha^{\prime} \sin \alpha+\sin \alpha\right)\right) \\
\sin \alpha & \frac{1}{1+\zeta}\left(\frac{R+u+w^{\prime}}{R}+\zeta\left(\cos \alpha+\alpha^{\prime} \cos \alpha\right)\right)
\end{array}\right)
$$

and developing $\frac{1}{1+\zeta}$ to the second order:

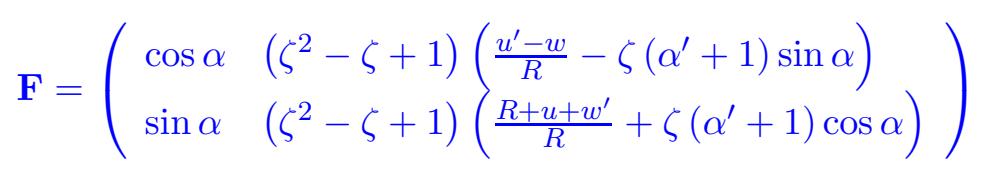

The strain tensor of the transformation is computed by:

$$
\mathbf{e}=\frac{1}{2}\left({ }^{T} \mathbf{F} . \mathbf{F}-\mathbf{I}\right)
$$

Detailing each component leads to:

$$
\begin{aligned}
e_{R R}= & 0 \\
e_{R \theta}= & e_{\theta R}=\frac{1}{2 R}\left(\zeta^{2}-\zeta+1\right)\left(\left(u^{\prime}-w\right) \cos \alpha+\left(R+u+w^{\prime}\right) \sin \alpha\right) \\
e_{\theta \theta}= & \frac{1-2 \zeta+3 \zeta^{2}}{2 R^{2}}\left(\left(u^{\prime}-w\right)^{2}+\left(R+u+w^{\prime}\right)^{2}\right. \\
& +2 R \zeta\left(\alpha^{\prime}+1\right)\left(\left(R+u+w^{\prime}\right) \cos \alpha-\left(u^{\prime}-w\right) \sin \alpha\right) \\
& \left.+R^{2} \zeta^{2}\left(\alpha^{\prime}+1\right)^{2}\right)-\frac{1}{2}
\end{aligned}
$$

\section{Remark:}

$e_{R R}=0$ means that there is no deformation along the direction $\mathbf{u}_{R}$ which is consistent with the hypothesis of thin beam.

\section{Appendix B. Internal forces}

The active forces on the cross section are the normal force $N$, the shear force $V$ and the moment $M$, given in the reference configuration $\left(\mathbf{u}_{R}, \mathbf{u}_{\theta}\right)$ by:

$$
\left\{\begin{array}{l}
N=\int_{A} S_{\theta \theta} d A \\
V=\int_{A} S_{R \theta} d A \\
M=\int_{A} z S_{\theta \theta} d A
\end{array}\right.
$$


with $A$ is the cross-section area. After calculation, the terms of force and moment in the local reference system are:

$$
\begin{aligned}
N= & \frac{E A}{2 R^{2}}\left(\left(u^{\prime}-w\right)^{2}+\left(R+u+w^{\prime}\right)^{2}-R^{2}\right) \\
& +\frac{3 E I}{2 R^{4}}\left(\left(u^{\prime}-w\right)^{2}+\left(R+u+w^{\prime}\right)^{2}\right) \\
& -\frac{2 E I}{R^{3}}\left(\alpha^{\prime}+1\right)\left(\left(R+u+w^{\prime}\right) \cos \alpha-\left(u^{\prime}-w\right) \sin \alpha\right)+\frac{E I}{2 R^{2}}\left(\alpha^{\prime}+1\right)^{2} \\
V= & \left(\frac{G A}{R}+\frac{G I}{R^{3}}\right)\left(\left(u^{\prime}-w\right) \cos \alpha+\left(R+u+w^{\prime}\right) \sin \alpha\right) \\
M= & -\frac{E I}{R^{3}}\left(\left(u^{\prime}-w\right)^{2}+\left(R+u+w^{\prime}\right)^{2}\right. \\
& \left.+\frac{E I}{R^{2}}\left(\alpha^{\prime}+1\right)\left(\left(R+u+w^{\prime}\right) \cos \alpha-\left(u^{\prime}-w\right) \sin \alpha\right)\right)
\end{aligned}
$$

with $I=\int_{A} z^{2} d A ; \int_{A} z d A=0$ et $A=\int_{A} d A$.

\section{Appendix C. Equilibrium of moment}

Proposition 1: for all vector a and second order tensor A defined in an Euclidian space on a domain $\mathfrak{D}$, we always have:

$$
\int_{\mathfrak{D}} \mathbf{a} \wedge \operatorname{div}(\mathbf{A}) d V=\int_{\partial \mathfrak{D}}(\mathbf{a} \wedge \mathbf{A} \cdot \mathbf{n}) d S+\int_{\mathfrak{D}} \mathbf{v} d V
$$

with $\mathbf{n}$ the normal vector to outside the boundary of $\Omega, \mathbf{v}$ a vector whose components are given by:

$\mathbf{v}=\left(\begin{array}{l}v_{1} \\ v_{2} \\ v_{3}\end{array}\right) ; \quad \operatorname{grad}(\mathbf{a}) \cdot \mathbf{A}^{T}-\mathbf{A} \cdot \operatorname{grad}(\mathbf{a})^{T}=\left(\begin{array}{ccc}0 & -v_{3} & v_{2} \\ & 0 & -v_{1} \\ \text { anti }- \text { sym } & 0\end{array}\right)$

Demonstration: As the gradient and divergence operations do not depend on the chosen reference system, to simplify the discussion, we can consider Cartesian coordinates. Writing in this basis, we get:

$$
\int_{\mathfrak{D}} \mathbf{a} \wedge \operatorname{div}(\mathbf{A}) d V=\int_{\mathfrak{D}}\left(a_{i} \mathbf{e}_{i}\right) \wedge\left(A_{i j, j} \mathbf{e}_{i}\right) d V
$$

with

$$
A_{i j, j}=\frac{\partial A_{i j}}{\partial x_{j}}
$$


So,

$$
\begin{aligned}
& \int_{\mathfrak{D}} \mathbf{a} \wedge \operatorname{div}(\mathbf{A}) d V=\int_{\mathfrak{D}}\left(\left(a_{2} A_{3 j, j}-a_{3} A_{2 j, j}\right) \mathbf{e}_{1}+\right. \\
&\left.\left(a_{3} A_{1 j, j}-a_{1} A_{3 j, j}\right) \mathbf{e}_{2}+\left(a_{1} A_{2 j, j}-a_{2} A_{1 j, j}\right) \mathbf{e}_{3}\right) d V
\end{aligned}
$$

Applying the Stokes' theorem, we obtain:

$$
\begin{aligned}
& \int_{\mathfrak{D}}\left(a_{2} A_{3 j, j}-a_{3} A_{2 j, j}\right) \mathbf{e}_{1} d V=\oint_{\partial \mathfrak{P}}\left(a_{2} A_{3 j} n_{j}-a_{3} A_{2 j} n_{j}\right) \mathbf{e}_{1} d S-\int_{\mathfrak{D}}\left(a_{2, j} A_{3 j}-a_{3, j} A_{2 j}\right) \mathbf{e}_{1} d V \\
& \int_{\mathfrak{D}}\left(a_{3} A_{1 j, j}-a_{1} A_{3 j, j}\right) \mathbf{e}_{2} d V=\oint_{\partial \mathfrak{D}}\left(a_{3} A_{1 j} n_{j}-a_{1} A_{3 j} n_{j}\right) \mathbf{e}_{2} d S-\int_{\mathcal{D}}\left(a_{3, j} A_{1 j}-a_{1, j} A_{3 j}\right) \mathbf{e}_{2} d V \\
& \int_{\mathfrak{D}}\left(a_{1} A_{2 j, j}-a_{2} A_{1 j, j}\right) \mathbf{e}_{3} d V=\oint_{\partial \mathfrak{D}}\left(a_{1} A_{2 j} n_{j}-a_{2} A_{1 j} n_{j}\right) \mathbf{e}_{3} d S-\int_{\mathfrak{D}}\left(a_{1, j} A_{2 j}-a_{2, j} A_{1 j}\right) \mathbf{e}_{3} d V
\end{aligned}
$$

with $n_{j}$ are the components of the normal vectors outward the domain $\mathfrak{D}$.

So,

$$
\begin{aligned}
\int_{\mathfrak{D}} \mathbf{a} \wedge & \operatorname{div}(\mathbf{A}) d V=\int_{\partial \mathfrak{D}}(\mathbf{a} \wedge \mathbf{A} \cdot \mathbf{n}) d S-\int_{\mathfrak{D}}\left(a_{2, j} A_{3 j}-a_{3, j} A_{2 j}\right) \mathbf{e}_{1} d V \\
& -\int_{\mathfrak{D}}\left(a_{3, j} A_{1 j}-a_{1, j} A_{3 j}\right) \mathbf{e}_{2} d V-\int_{\mathfrak{D}}\left(a_{1, j} A_{2 j}-a_{2, j} A_{1 j}\right) \mathbf{e}_{3} d V
\end{aligned}
$$

If we define:

$$
\mathbf{v}=\left(\begin{array}{c}
v_{1} \\
v_{2} \\
v_{3}
\end{array}\right)=\left(\begin{array}{c}
a_{3, j} A_{2 j}-a_{2, j} A_{3 j} \\
a_{1, j} A_{3 j}-a_{3, j} A_{1 j} \\
a_{2, j} A_{1 j}-a_{1, j} A_{2 j}
\end{array}\right)
$$

Otherwise:

$$
\operatorname{grad}(\mathbf{a}) \cdot \mathbf{A}^{T}-\mathbf{A} \cdot \operatorname{grad}(\mathbf{a})^{T}=\left(a_{i, k} A_{j k}-A_{i k} a_{j, k}\right) \mathbf{e}_{i} \otimes \mathbf{e}_{j}
$$

under a matrix form:

$$
\operatorname{grad}(\mathbf{a}) \cdot \mathbf{A}^{T}-\mathbf{A} \cdot \operatorname{grad}(\mathbf{a})^{T}=\left(\begin{array}{ccc}
0 & -v_{3} & v_{2} \\
& 0 & -v_{1} \\
\text { skewsym } & & 0
\end{array}\right)
$$

we get:

$$
\int_{\mathfrak{D}} \mathbf{a} \wedge \operatorname{div}(\mathbf{A}) d V=\int_{\partial \mathfrak{D}}(\mathbf{a} \wedge \mathbf{A} \cdot \mathbf{n}) d S+\int_{\mathfrak{D}} \mathbf{v} d V
$$

We apply this relation in (50) and we get:

$$
\int_{\mathfrak{D}} \mathbf{S}^{\prime} \mathbf{P}^{\prime} \wedge \operatorname{div}(\mathbf{P}) d V=\oint_{\partial \mathcal{D}}\left(\mathbf{S}^{\prime} \mathbf{P}^{\prime} \wedge \mathbf{P} . \mathbf{n}\right) d V+\int_{\mathfrak{D}} \mathbf{v} d V
$$


and

$$
\begin{aligned}
& \oint_{\partial \mathfrak{D}}\left(\mathbf{S}^{\prime} \mathbf{P}^{\prime} \wedge \mathbf{P} . \mathbf{n}\right) d V=\int_{A_{s+d s}}\left(\mathbf{S}^{\prime} \mathbf{P}^{\prime} \wedge \mathbf{P}_{s+d s} . \mathbf{s}\right) d A-\int_{A_{s}}\left(\mathbf{S}^{\prime} \mathbf{P}^{\prime} \wedge \mathbf{P}_{s} . \mathbf{s}\right) d A+ \\
& +\int_{\partial \mathfrak{D}_{\text {lateral }}}\left(\mathbf{S}^{\prime} \mathbf{P}^{\prime} \wedge \mathbf{P} . \mathbf{n}\right) d A=\mathbf{M}_{s+d s}-\mathbf{M}_{s}+d s \oint_{\partial A}\left(\mathbf{S}^{\prime} \mathbf{P}^{\prime} \wedge \mathbf{P} . \mathbf{n}\right) d l \quad(\text { C.3 })
\end{aligned}
$$

with $\mathbf{M}_{s+d s}$ and $\mathbf{M}_{s}$ are the moments on the right section respectively at the positions $(s+d s)$ and $(s)$. By definition:

$$
\begin{gathered}
\int_{A_{s+d s}}\left(\mathbf{S}^{\prime} \mathbf{P}^{\prime} \wedge \mathbf{P}_{s+d s} \cdot \mathbf{s}\right) d A=\mathbf{M}_{s+d s} \\
\int_{A_{s}}\left(\mathbf{S}^{\prime} \mathbf{P}^{\prime} \wedge \mathbf{P}_{s} \cdot \mathbf{s}\right) d A=\mathbf{M}_{s}
\end{gathered}
$$

The components of the vector $\mathbf{v}$ are calculated in equation (C.2):

$$
\begin{aligned}
& \operatorname{grad}\left(\mathbf{S}^{\prime} \mathbf{P}^{\prime}\right) \cdot \mathbf{P}^{T}-\mathbf{P} \cdot \operatorname{grad}\left(\mathbf{S}^{\prime} \mathbf{P}^{\prime}\right)^{T} \\
= & \operatorname{grad}\left(\mathbf{O P}^{\prime}-\mathbf{O S}^{\prime}\right) \cdot \mathbf{P}^{T}-\mathbf{P} \cdot \operatorname{grad}\left(\mathbf{O P}^{\prime}-\mathbf{O S}^{\prime}\right)^{T} \\
= & \operatorname{grad}\left(\mathbf{O P}^{\prime}\right) \cdot \mathbf{P}^{T}-\mathbf{P} \cdot \operatorname{grad}\left(\mathbf{O P}^{\prime}\right)^{T}-\left(\operatorname{grad}\left(\mathbf{O S}^{\prime}\right) \cdot \mathbf{P}^{T}-\mathbf{P} \cdot \operatorname{grad}\left(\mathbf{O S}^{\prime}\right)^{T}\right) \\
= & \mathbf{F} \cdot \mathbf{P}^{T}-\mathbf{P} \cdot \mathbf{F}^{T}-\left(\operatorname{grad}\left(\mathbf{O} \mathbf{S}^{\prime}\right) \cdot \mathbf{P}^{T}-\mathbf{P} \cdot \operatorname{grad}\left(\mathbf{O S}^{\prime}\right)^{T}\right)
\end{aligned}
$$

As the second Piola-Kirchhoff stress tensor is always symmetric, we have:

$$
\mathbf{S}=\mathbf{S}^{T} \rightarrow \mathbf{F} . \mathbf{S} . \mathbf{F}^{T}=\mathbf{F} . \mathbf{S}^{T} . \mathbf{F}^{T} \rightarrow \mathbf{P} . \mathbf{F}^{T}=\mathbf{F} . \mathbf{P}^{T} \rightarrow \mathbf{F} . \mathbf{P}^{T}-\mathbf{P} . \mathbf{F}^{T}=\mathbf{0}
$$

So,

$$
\begin{gathered}
\operatorname{grad}\left(\mathbf{S}^{\prime} \mathbf{P}^{\prime}\right) \cdot \mathbf{P}^{T}-\mathbf{P} \cdot \operatorname{grad}\left(\mathbf{S}^{\prime} \mathbf{P}^{\prime}\right)^{T}=-\left(\operatorname{grad}\left(\mathbf{O S}^{\prime}\right) \cdot \mathbf{P}^{T}-\mathbf{P} \cdot \operatorname{grad}\left(\mathbf{O S}^{\prime}\right)^{T}\right) \\
=-\left(\left(\frac{\partial\left(\mathbf{O} \mathbf{S}^{\prime}\right)}{\partial s} \otimes \mathbf{s}\right) \cdot\left(\mathbf{P}_{i} \otimes \mathbf{s}_{i}+\mathbf{P}_{3} \otimes \mathbf{s}\right)^{T}-\left(\mathbf{P}_{i} \otimes \mathbf{s}_{i}+\mathbf{P}_{3} \otimes \mathbf{s}\right) \cdot\left(\frac{\partial\left(\mathbf{O} \mathbf{S}^{\prime}\right)}{\partial s} \otimes \mathbf{s}\right)^{T}\right) \\
=-\left(\left(\frac{\partial\left(\mathbf{O} \mathbf{S}^{\prime}\right)}{\partial s} \otimes \mathbf{P}_{3}\right)-\left(\mathbf{P}_{3} \otimes \frac{\partial\left(\mathbf{O} \mathbf{S}^{\prime}\right)}{\partial s}\right)\right)
\end{gathered}
$$

By the definition of the vector $\mathbf{v}$ in the proposal, we find that:

$$
\mathbf{v}=\frac{\partial\left(\mathbf{O S}^{\prime}\right)}{\partial s} \wedge \mathbf{P}_{3}
$$


Combining (C.2), (C.3) and (C.5) :

$$
\begin{gathered}
\int_{\mathcal{D}} \mathbf{S}^{\prime} \mathbf{P}^{\prime} \wedge \operatorname{div}(\mathbf{P}) d V=\mathbf{M}_{s+d s}-\mathbf{M}_{s}+d s \oint_{\partial A}\left(\mathbf{S}^{\prime} \mathbf{P}^{\prime} \wedge \mathbf{P} . \mathbf{n}\right) d l+\int_{\mathcal{D}} \frac{\partial\left(\mathbf{O S}^{\prime}\right)}{\partial s} \wedge \mathbf{P}_{3} d V \\
=\mathbf{M}_{s+d s}-\mathbf{M}_{s}+d s \oint_{\partial A}\left(\mathbf{S}^{\prime} \mathbf{P}^{\prime} \wedge \mathbf{q}^{s}\right) d l+d s \int_{A} \frac{\partial\left(\mathbf{O S}^{\prime}\right)}{\partial s} \wedge \mathbf{P}_{3} d A \\
=\mathbf{M}_{s+d s}-\mathbf{M}_{s}+d s \oint_{\partial A}\left(\mathbf{S}^{\prime} \mathbf{P}^{\prime} \wedge \mathbf{q}^{s}\right) d l+d s \frac{\partial\left(\mathbf{O S}^{\prime}\right)}{\partial s} \wedge \int_{A} \mathbf{P}_{3} d A \\
=\mathbf{M}_{s+d s}-\mathbf{M}_{s}+d s \oint_{\partial A}\left(\mathbf{S}^{\prime} \mathbf{P}^{\prime} \wedge \mathbf{q}^{s}\right) d l+d s \frac{\partial\left(\mathbf{O} \mathbf{S}^{\prime}\right)}{\partial s} \wedge \mathbf{f} \quad \text { (C.6) }
\end{gathered}
$$

From (50), (51) and (C.6), we get:

$$
\begin{aligned}
\int_{A} \rho \mathbf{S}^{\prime} \mathbf{P}^{\prime} \wedge \operatorname{grad}(\mathbf{F} \cdot \boldsymbol{\Omega}(\mathbf{x}, t)) \cdot \boldsymbol{\Omega}(\mathbf{x}, t) d A \\
\quad=\int_{A} \mathbf{S}^{\prime} \mathbf{P}^{\prime} \wedge \mathbf{q}^{v} d A+\frac{\mathbf{M}_{s+d s}-\mathbf{M}_{s}}{d s}+\oint_{\partial A}\left(\mathbf{S}^{\prime} \mathbf{P}^{\prime} \wedge \mathbf{q}^{s}\right) d l+\frac{\partial\left(\mathbf{O S}^{\prime}\right)}{\partial s} \wedge \mathbf{f}
\end{aligned}
$$

We get the equilibrium equation:

$$
\begin{aligned}
\frac{\partial \mathbf{M}}{\partial s}+\frac{\partial\left(\mathbf{O S}^{\prime}\right)}{\partial s} \wedge \mathbf{f}+\int_{A} \mathbf{S}^{\prime} \mathbf{P}^{\prime} \wedge \mathbf{q}^{v} d A \\
-\int_{A} \rho \mathbf{S}^{\prime} \mathbf{P}^{\prime} \wedge \operatorname{grad}(\mathbf{F} \cdot \boldsymbol{\Omega}(\mathbf{x}, t)) \cdot \boldsymbol{\Omega}(\mathbf{x}, t) d A+\oint_{\partial A}\left(\mathbf{S}^{\prime} \mathbf{P}^{\prime} \wedge \mathbf{q}^{s}\right) d l=0
\end{aligned}
$$

\section{Appendix D. Body forces}

Returning to the expression of the gradient tensor of the transformation given by (A.3), one gets:

$$
\begin{aligned}
\mathbf{F} . \boldsymbol{\Omega}(\mathbf{X}, t) & =\left(\begin{array}{cc}
\cos \alpha & \frac{1}{R+z}\left(u^{\prime}-w-z\left(\alpha^{\prime}+1\right) \sin \alpha\right) \\
\sin \alpha & \frac{1}{R+z}\left(R+w^{\prime}+u+z\left(\alpha^{\prime}+1\right) \cos \alpha\right)
\end{array}\right) \Omega\left(\begin{array}{c}
0 \\
R+z
\end{array}\right) \\
& =\Omega\left(\begin{array}{c}
u^{\prime}-w-z\left(\alpha^{\prime}+1\right) \sin \alpha \\
R+w^{\prime}+u+z\left(\alpha^{\prime}+1\right) \cos \alpha
\end{array}\right)
\end{aligned}
$$

Taking equation (D.1) :

$$
\begin{aligned}
& d(\mathbf{F} . \boldsymbol{\Omega})=\Omega\left(\left(-\left(\alpha^{\prime}+1\right) \sin \alpha d z+\left(u^{\prime \prime}-w^{\prime}-z\left(\alpha^{\prime \prime} \sin \alpha+\left(\alpha^{\prime}+1\right) \alpha^{\prime} \cos \alpha\right)\right) d \theta\right) \mathbf{u}_{R}\right. \\
& +\left(u^{\prime}-w-z\left(\alpha^{\prime}+1\right) \sin \alpha\right) d \theta \mathbf{u}_{\theta}-\left(R+w^{\prime}+u+z\left(\alpha^{\prime}+1\right) \cos \alpha\right) d \theta \mathbf{u}_{R} \\
& \left.\quad\left(\left(\alpha^{\prime}+1\right) \cos \alpha d z+\left(w^{\prime \prime}+u^{\prime}+z\left(\alpha^{\prime \prime} \cos \alpha-\left(\alpha^{\prime}+1\right) \alpha^{\prime} \sin \alpha\right)\right) d \theta\right) \mathbf{u}_{\theta}\right)
\end{aligned}
$$


Replacing in relation (A.1), one gets

$$
\begin{aligned}
& \operatorname{grad}(\mathbf{F} . \boldsymbol{\Omega})=\Omega\left(-\left(\alpha^{\prime}+1\right) \sin \alpha \mathbf{u}_{R} \otimes \mathbf{u}_{R}+\right. \\
& \frac{1}{R+z}\left(-R+u^{\prime \prime}-2 w^{\prime}-u-z\left(\alpha^{\prime \prime} \sin \alpha+\left(\alpha^{\prime}+1\right)^{2} \cos \alpha\right)\right) \mathbf{u}_{R} \otimes \mathbf{u}_{\theta}+\left(\alpha^{\prime}+1\right) \cos \alpha \mathbf{u}_{\theta} \otimes \mathbf{u}_{R} \\
& \left.\frac{1}{R+z}\left(w^{\prime \prime}+2 u^{\prime}-w-z\left(-\alpha^{\prime \prime} \cos \alpha+\left(\alpha^{\prime}+1\right)^{2} \sin \alpha\right)\right) \mathbf{u}_{\theta} \otimes \mathbf{u}_{\theta}\right) \quad \text { (D.3) }
\end{aligned}
$$

Finally,

$$
\begin{array}{r}
\operatorname{grad}(\mathbf{F} . \boldsymbol{\Omega}) . \boldsymbol{\Omega}=\Omega^{2}\left(\left(-R+u^{\prime \prime}-2 w^{\prime}-u-z\left(\alpha^{\prime \prime} \sin \alpha+\left(\alpha^{\prime}+1\right)^{2} \cos \alpha\right)\right) \mathbf{u}_{R}\right. \\
\left.+\left(w^{\prime \prime}+2 u^{\prime}-w-z\left(-\alpha^{\prime \prime} \cos \alpha+\left(\alpha^{\prime}+1\right)^{2} \sin \alpha\right)\right) \mathbf{u}_{\theta}\right) \quad(\text { D. } 4)
\end{array}
$$

which can also be written as:

$$
\begin{aligned}
\operatorname{grad} & (\mathbf{F} \cdot \boldsymbol{\Omega}(\mathbf{X}, t)) \cdot \boldsymbol{\Omega}(\mathbf{X}, t) \\
= & \Omega^{2}\left(\begin{array}{c}
-R+u^{\prime \prime}-2 w^{\prime}-u-z\left(\cos (\alpha)\left(\alpha^{\prime}+1\right)^{2}+\alpha^{\prime \prime} \sin (\alpha)\right) \\
w^{\prime \prime}+2 u^{\prime}-w-z\left(\sin (\alpha)\left(\alpha^{\prime}+1\right)^{2}-\alpha^{\prime \prime} \cos (\alpha)\right)
\end{array}\right)
\end{aligned}
$$

The body force is then given by integrating over the cross section in the reference system $\left(\mathbf{u}_{R}, \mathbf{u}_{\theta}\right)$ :

$$
-\rho \int_{A} \operatorname{grad}(\mathbf{F} \boldsymbol{\Omega}(\mathbf{X}, t)) \cdot \boldsymbol{\Omega}(\mathbf{X}, t) d A=-\rho \Omega^{2} A\left(\begin{array}{c}
-R+u^{\prime \prime}-2 w^{\prime}-u \\
w^{\prime \prime}+2 u^{\prime}-w
\end{array}\right)
$$

\section{Appendix E. Dynamic forces}

Neglecting all second order terms in $\mathbf{u}_{t}$ the dynamic forces are computed from (B.2) by: 


$$
\begin{aligned}
N_{t}= & \left(\frac{E A}{R^{2}}+\frac{3 E I}{R^{4}}\right)\left(\left(u^{\prime}-w\right)\left(u_{t}^{\prime}-w_{t}\right)+\left(u_{t}+w_{t}^{\prime}\right)\left(R+u+w^{\prime}\right)\right) \\
& +\frac{E I}{R^{2}} \alpha_{t}^{\prime}\left(\alpha^{\prime}+1\right)-\frac{2 E I}{R^{3}} \alpha_{t}^{\prime}\left(\left(R+u+w^{\prime}\right) \cos \alpha-\left(u^{\prime}-w\right) \sin \alpha\right) \\
& -\frac{2 E I}{R^{3}}\left(\alpha^{\prime}+1\right)\left(\left(u_{t}+w_{t}^{\prime}\right) \cos \alpha-\left(u_{t}^{\prime}-w_{t}\right) \sin \alpha\right. \\
& \left.-\alpha_{t}\left(\left(R+u+w^{\prime}\right) \sin \alpha+\left(u^{\prime}-w\right) \cos \alpha\right)\right) \\
V_{t}= & \left(\frac{G A}{R}+\frac{G I}{R^{3}}\right)\left(\left(u_{t}^{\prime}-w_{t}\right) \cos \alpha+\left(u_{t}+w_{t}^{\prime}\right) \sin \alpha\right) \\
& +\left(\frac{G A}{R}+\frac{G I}{R^{3}}\right) \alpha_{t}\left(\left(R+u+w^{\prime}\right) \cos \alpha-\left(u^{\prime}-w\right) \sin \alpha\right) \\
M_{t}= & -\frac{2 E I}{R^{3}}\left(\left(u_{t}^{\prime}-w_{t}\right)\left(u^{\prime}-w\right)+\left(u_{t}+w_{t}^{\prime}\right)\left(R+u+w^{\prime}\right)\right) \\
& +\frac{E I}{R^{2}} \alpha_{t}^{\prime}\left(\left(R+u+w^{\prime}\right) \cos \alpha-\left(u^{\prime}-w\right) \sin \alpha\right) \\
& +\frac{E I}{R^{2}}\left(\alpha^{\prime}+1\right)\left(\left(u_{t}+w_{t}^{\prime}\right) \cos \alpha-\left(u_{t}^{\prime}-w_{t}\right) \sin \alpha\right. \\
& \left.-\alpha_{t}\left(\left(R+u+w^{\prime}\right) \sin \alpha+\left(u^{\prime}-w\right) \cos \alpha\right)\right)
\end{aligned}
$$

The linearized balance equations of the vector of displacements $\left(u_{t}, w_{t}, \alpha_{t}\right)$ are obtained by subtracting the stationary part of equations (30) and (69) in the same way that in the linear case. The terms of second order in $\left(u_{t}, w_{t}, \alpha_{t}\right)$ are neglected and the dynamic equations are in the basis $\left(\mathbf{u}_{R}, \mathbf{u}_{\theta}\right)$ :

$$
\begin{aligned}
& \frac{1}{R}\left(V_{t}^{\prime} \cos \alpha-N_{t}^{\prime} \sin \alpha-\alpha_{t}^{\prime}(V \sin \alpha+N \cos \alpha)\right)+p \frac{u_{t}+w_{t}^{\prime}}{R}+p_{t} \frac{R+u+w^{\prime}}{R} \\
& +\frac{1}{R}\left(-\alpha_{t}\left(V^{\prime} \sin \alpha+N^{\prime} \cos \alpha\right)-\left(\alpha^{\prime}+1\right)\left(V_{t} \sin \alpha+N_{t} \cos \alpha\right.\right. \\
& \left.\left.+\alpha_{t}(V \cos \alpha-N \sin \alpha)\right)\right)+f_{R}{ }^{t}-k_{R} u_{t}=\rho A \ddot{u}_{t} \\
& \frac{1}{R}\left(V_{t}^{\prime} \sin \alpha+N_{t}^{\prime} \cos \alpha+\alpha_{t}^{\prime}(V \cos \alpha-N \sin \alpha)\right)-p \frac{u_{t}^{\prime}-w_{t}}{R}-p_{t} \frac{u^{\prime}-w}{R} \\
& +\frac{1}{R}\left(\alpha_{t}\left(V^{\prime} \cos \alpha-N^{\prime} \sin \alpha\right)+\left(\alpha^{\prime}+1\right)\left(V_{t} \cos \alpha-N_{t} \sin \alpha\right.\right. \\
& \left.\left.-\alpha_{t}(N \cos \alpha+V \sin \alpha)\right)\right)-k_{\theta} w_{t}=\rho A \ddot{w}_{t} \\
& \frac{1}{R} M_{t}^{\prime}+\frac{1}{R}\left(\left(u_{t}^{\prime}-w_{t}\right)(V \sin \alpha+N \cos \alpha)-\left(u_{t}+w_{t}^{\prime}\right)(V \cos \alpha-N \sin \alpha)\right) \\
& +\frac{1}{R}\left(\left(u^{\prime}-w\right)\left(V_{t} \sin \alpha+N_{t} \cos \alpha\right)-\left(R+u+w^{\prime}\right)\left(V_{t} \cos \alpha-N_{t} \sin \alpha\right)\right) \\
& +\frac{1}{R}\left(\alpha_{t}\left(u^{\prime}-w\right)(V \cos \alpha-N \sin \alpha)+\alpha_{t}\left(R+u+w^{\prime}\right)(V \sin \alpha+N \cos \alpha)\right)=\rho I \ddot{\alpha}_{t}
\end{aligned}
$$




\section{References}

[1] F. Böhm. Mechanik des gürtelreifens. Ingenieur-Archiv, 35(2):82-101, 1966.

[2] M. Heckl. Tyre noise generation. Wear, 113(1):157-170, 1986.

[3] W. Kropp. Structure-borne sound on a smooth tyre. Applied Acoustics, 26(3):181-192, 1989.

[4] J. Perisse and J.F. Hamet. A comparison of the $2 \mathrm{~d}$ ring and $3 \mathrm{~d}$ orthotropic plate for modelling of radial tire vibrations. In INTERNOISE 2000, Nice, France, August 27-30 2000.

[5] R. Meftah. Une approche par formalisme de green réduit pour le calcul des structures en contact dynamique: application au contact pneumatique/chaussée. PhD thesis, Université Paris-Est, 2011.

[6] J. Périsse. A study of radial vibrations of a rolling tyre for tyreroad noise characterisation. Mechanical systems and signal processing, 16(6):1043-1058, 2002.

[7] P.H. Campanac, D. Duhamel, and K. Nonami. Modeling of tire vibrations. In INTER-NOISE 99, Fort Lauderdale, Florida, USA, December, 06-08 1999.

[8] P.H. Campanac, K. Nonami, and D. Duhamel. Application of the vibration analysis of linear systems with time periodic coefficients to the dynamics of a rolling tyre. Journal of sound and vibration, 231(1):3777,2000 .

[9] Y.J. Kim and J.S. Bolton. Modeling tire treadband vibration. In InterNoise 2001, The Hague, Netherlands, August 2001.

[10] S.C. Huang and B.S. Hsu. An approach to the dynamic analysis of rotating tire wheel suspension units. Journal of sound and vibration, 156(3):505-519, 1992.

[11] M. Eichler. A ride comfort tyre model for vibration analysis in full vehicle simulations. Vehicle System Dynamics, 27(S1):109-122, 1997.

[12] M. Gipser. Ftire, a new fast tire model for ride comfort simulations. In International ADAMS User's Conference Berlin, Germany, pages 1-11, 1999.

[13] J.M. Muggleton, B.R. Mace, and M.J. Brennan. Vibrational response prediction of a pneumatic tyre using an orthotropic two-plate wave model. Journal of sound and vibration, 264(4):929-950, 2003. 
[14] J.F. Hamet. Tire/road noise: time domain green's function for the orthotropic plate model. Acta Acustica united with Acustica, 87(4):470474, 2001.

[15] R.J. Pinnington. A wave model of a circular tyre. In Proceedings of Forum Acusticum, Sevilla, Spain, September, 16-20 2002.

[16] R.J. Pinnington. A wave model of a circular tyre. part 1: belt modelling. Journal of Sound and Vibration, 290(1):101-132, 2006.

[17] R.J. Pinnington. A wave model of a circular tyre. part 2: side-wall and force transmission modelling. Journal of sound and vibration, 290(1):133-168, 2006.

[18] Y.J. Kim and J.S. Bolton. Effects of rotation on the dynamics of a circular cylindrical shell with application to tire vibration. Journal of sound and vibration, 275(3):605-621, 2004.

[19] F. Wullens and W. Kropp. Wave content of the vibration field of a rolling tyre. Acta Acustica united with Acustica, 93(1):48-56, 2007.

[20] R. Takagi and S. Takanari. Tire structural parameter analysis for road noise using an accurate fem model. SAE Technical Paper 911873, 1991.

[21] K.V. Narasimha Rao and R.K. Kumar. Simulation of tire dynamic behavior using various finite element techniques. International Journal for Computational Methods in Engineering Science and Mechanics, 8(5):363-372, 2007.

[22] T.L. Richards. Finite element analysis of structural-acoustic coupling in tyres. Journal of sound and vibration, 149(2):235-243, 1991.

[23] J.R. Cho, K.W. Kim, W.S. Yoo, and S.I. Hong. Mesh generation considering detailed tread blocks for reliable $3 \mathrm{~d}$ tire analysis. Advances in engineering software, 35(2):105-113, 2004.

[24] A. Fadavi. Modélisation numérique des vibrations d'un pneumatique et de la propagation du bruit de contact. $\mathrm{PhD}$ thesis, Ecole Nationale des Ponts et Chaussées, 2002.

[25] M. Brinkmeier, U. Nackenhorst, S. Petersen, and O. Von Estorff. A finite element approach for the simulation of tire rolling noise. Journal of Sound and Vibration, 309(1):20-39, 2008.

[26] Waki Y., Mace B.R., and Brennan M.J. Free and forced vibrations of a tyre using a wave/finite element approach. Journal of Sound and Vibration, 323(3):737-756, 2009. 
[27] D. Duhamel. A recursive approach for the finite element computation of waveguides. Journal of Sound and Vibration, 323(1):163-172, 2009.

[28] D. Duhamel, S. Erlicher, and H.H. Nguyen. A recursive finite element method for computing tyre vibrations. European Journal of Computational Mechanics, 20(1-4):9-27, 2011.

[29] P. Kindt, P. Sas, and W. Desmet. Development and validation of a three-dimensional ring-based structural tyre model. Journal of Sound and Vibration, 326(3-5):852-869, 2009.

[30] P. Kindt, P. Sas, and W. Desmet. Measurement and analysis of rolling tire vibrations. Optics and Lasers in Engineering, 47(3-4):443-453, 2009 .

[31] Z.C. Feng and P. Gu. Modeling and experimental verification of vibration and noise caused by the cavity modes of a rolling tire under static loading. SAE Technical Paper 2011-01-1581, 2011.

[32] M. Brinkmeier and U. Nackenhorst. An approach for large-scale gyroscopic eigenvalue problems with application to high-frequency response of rolling tyres. Computational Mechanics, 41(4):503-515, 2008.

[33] I. Lopez, R.E.A. Blom, N.B. Roozen, and H. Nijmeijer. Modelling vibrations on deformed rolling tyres - a modal approach. Journal of Sound and Vibration, 307(3-5):481-494, 2007.

[34] I. Lopez, R.R.J.J. van Doorn, R. van der Steen, N.B. Roozen, and H. Nijmeijer. Frequency loci veering due to deformation in rotating tyres. Journal of Sound and Vibration, 324(3-5):622-639, 2009.

[35] S.S. Antman. The theory of rods. In C. Truesdell, editor, Handbuch der Physik, volume VIa/2, pages 641-703. Springer-Verlag, 1972.

[36] S.S. Antman. Kirchhoff's problem for nonlinearly elastic rods. Quarterly of applied mathematics, 32:221-240, 1974.

[37] S.S. Antman. Bifurcation problems for nonlinearly elastic structures. In P.H. Rabinowitz, editor, Symposium on Applications of Bifurcation Theory, pages 73-125, New York, 1977. Academic Press.

[38] S.S. Antman. Nonlinear problems of elasticity. Springer, 2005.

[39] S.S. Antman and K.B. Jordan. Qualitative aspects of the spatial deformation of non-linearly elastic rods. Proceedings of the Royal Society of Edinburgh: Section A Mathematics, 73(1):85-105, 1975.

[40] M.M. Attard. Finite strain-beam theory. International journal of solids and structures, 40(17):4563-4584, 2003. 
[41] A.E. Green. The equilibrium of rods. Archive for Rational Mechanics and Analysis, 3(1):417-421, 1959.

[42] A.D. Lanzo. Nonlinear analysis of elastic high shear deformable plane frames by a mixed fem path following approach. International Journal of Solids and Structures, 46(7):1764-1771, 2009.

[43] M. Pignataro, A. Di Carlo, and R. Casciaro. On nonlinear beam models from the point of view of computational post buckling analysis. International Journal of Solids and Structures, 18(4):327-347, 1982.

[44] J.C. Simo. A finite strain beam formulation. the three dimensional dynamic problem. part i. Computer methods in applied mechanics and engineering, 49(1):55-70, 1985.

[45] J.C. Simo and L. Vu-Quoc. On the dynamics of flexible beams under large overall motions. the plane case. i, ii. ASME, Transactions, Journal of Applied Mechanics, 53(4):849-863, 1986.

[46] J.C. Simo and L. Vu-Quoc. A three dimensional finite strain rod model. part ii: Computational aspects. Computer methods in applied mechanics and engineering, 58(1):79-116, 1986.

[47] F. Davi. The theory of Kirchhoff rods as an exact consequence of three dimensional elasticity. Journal of elasticity, 29(3):243-262, 1992.

[48] A.D. Lanzo. On elastic beam models for stability analysis of multilayered rubber bearings. International journal of solids and structures, 41(20):5733-5757, 2004.

[49] U. Nackenhorst. The ALE-formulation of bodies in rolling contact: Theoretical foundations and finite element approach. Computer Methods in Applied Mechanics and Engineering, 193(39-41):4299-4322, 2004.

[50] C. Bacon and J. Pouyet. Mécanique des solides déformables. Hermés science, 2000.

[51] J.-P. Ponthot. Traitement unifié de la Mécanique des Milieux Continus solides en grandes transformations par la méthode des éléments finis. Collection des publications de la faculté des Sciences Appliquées, N 159, Université de Liége, 1995. 